\title{
ZUM WIRKUNGSKREIS EINER KLEINASIATISCHEN MATRONA ANHAND AUSGEWÄHLTER FUNDE AUS DEM HANGHAUS 2 IN EPHESOS*
}

\author{
Einleitung
}

»To read the fragmentary information about women in public space as exceptions and anomalies; to write about women only in the context of the household space or the brothel; or to investigate the household space as the space of women, is to adopt the world view and filtering gaze of the ancient public male. $\ll^{1}$

Nicht immer treffen dieselben glücklich-unglücklichen Umstände zu, in denen ein Bauwerk oder eine Anlage 'mitten im Leben' zerstört wird wie in Pompeji. Deshalb sind auch die Funde aus der römischen Kaiserzeit, die in den 'reichen' Hanghäusern in Ephesos gemacht wurden, sicherlich nur bedingt aussagekräftig. Dies gilt sogar für Artefakte, die beispielsweise in einer Brandschicht bzw. direkt auf einem Boden gefunden wurden, denn die Bau- bzw. besser gesagt die Zerstörungsgeschichte des Hanghauses 2 zeigt, daß sich die Wohnungen zum Zeitpunkt der Aufgabe teilweise in Reparatur befanden und nicht wie in Pompeji und Herkulaneum durch die Zerstörung direkt aus dem Leben gerissen worden waren. Daher ist bei dem Fundmaterial keineswegs mit Vollständigkeit zu rechnen².

Dennoch sollte uns dieser Umstand nicht von dem Versuch abhalten, das vorhandene Material entsprechend auszuwerten - die individuellen Rahmenbedingungen dürfen dabei allerdings nicht außer acht gelassen werden -, obgleich diese Vorgangsweise in jüngster Zeit scharf unter Beschuß gerät und ihre Zulässigkeit sogar für wirkliche Zerstörungsbefunde wie jene der Vesuvstädte in Abrede gestellt wird ${ }^{3}$. Es ist sicherlich richtig, die Multifunktionalität der Räume in

* Die Beschäftigung mit dieser Fundgruppe wurde durch die Finanzierung der Österreichischen Nationalbank (P 9004) ermöglicht und erfolgte am Institut für Kulturgeschichte der Antike, ÖAW, unter der Leitung von H. Thür. K. Koller, S. Ladstätter, E. Rathmayr und N. Zimmermann unterstützten mich beim Verständnis der Fundumstände. Darüber hinaus schulde ich allen Kolleg(inn)en, die mit der Bearbeitung von Befunden und Funden aus den beiden Hanghäusern beschäftigt sind, meinen Dank. Den Mitarbeiter(inne)n des Efes Müzesi in Selçuk danke ich für die Zusammenarbeit. Mein Dank ergeht außerdem an die Grabungsleitung Ephesos, mit deren Erlaubnis ich die Spinnrocken von den Grabungen am Staatsmarkt und am Magnesischen Tor an dieser Stelle anschließen darf. V. Mitsopoulos-Leon und G. Seiterle danke ich in diesem Zusammenhang für bereitwillige Unterstützung. - Die hier zusätzlich verwendeten Abkürzungen finden sich am Ende des Beitrags.

1 J. Okland, In publicum procurrendi: Women in the Public Space of Roman Greece, in: Larrson Lovén - Strömberg, Women 139. Vgl. auch E. Scott, Women and Gender Relations in the Roman Empire, in: P. Rush (Hrsg.), Theoretical Roman Archaeology: Second Conference Proceedings (1995) 174-189. Dickmann, Domus 377: »Wenn in der vorliegenden Arbeit Wohnvorgänge fast ausschließlich aus der Sicht des dominus rekonstruiert werden konnten, dann hängt dies exakt mit den Aussagen der Quellen einerseits und der alleinigen Erhaltung der räumlichen Strukturen andererseits zusammen.«

2 Beispielsweise fehlt Tafelgeschirr aus Edelmetall nahezu gänzlich, obwohl ein größerer römischer Haushalt zweifelsohne solches besaß; Petron. 31 ff. - Zur Bau- und Zerstörungsgeschichte vgl. bes. die Einzelstudien von S. Ladstätter, H. Thür und G. Wiplinger in: Krinzinger, Baugeschichte.

3 B. Sigges, vita cognita. Die Ausstattung pompejianischer Wohnhäuser mit Gefäßen und Geräten - untersucht an ausgewählten Beispielen (ungedr. Diss. Köln 2000) bes. 71 ff. 


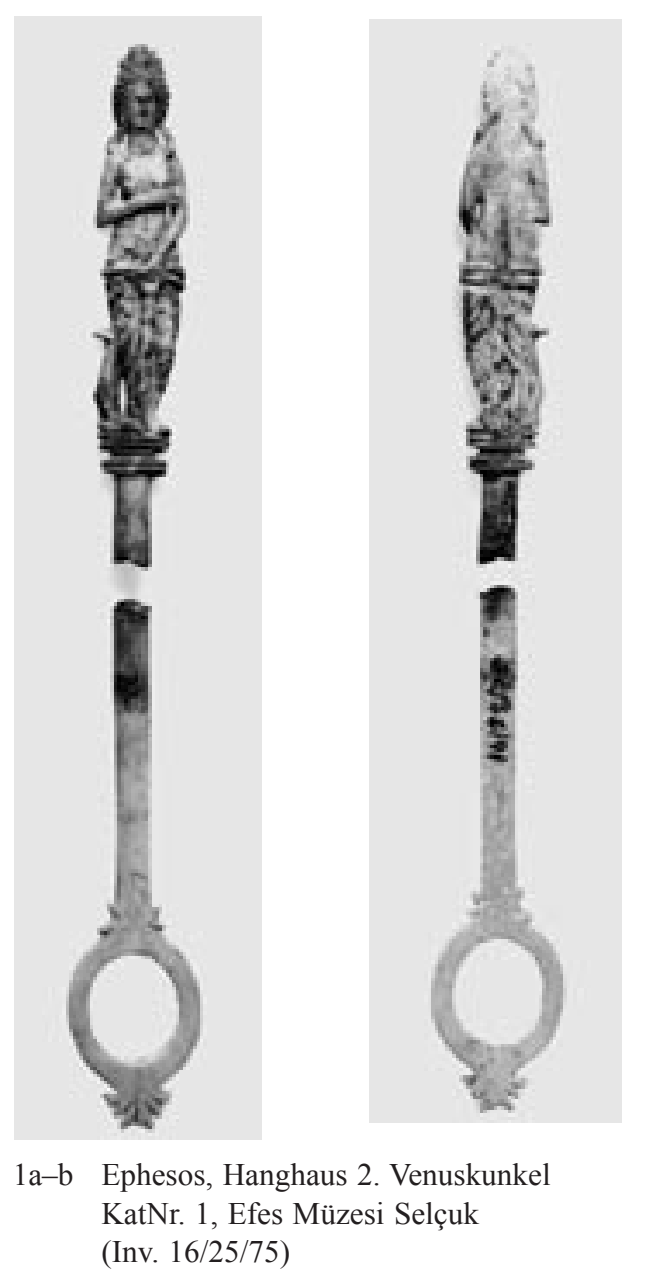

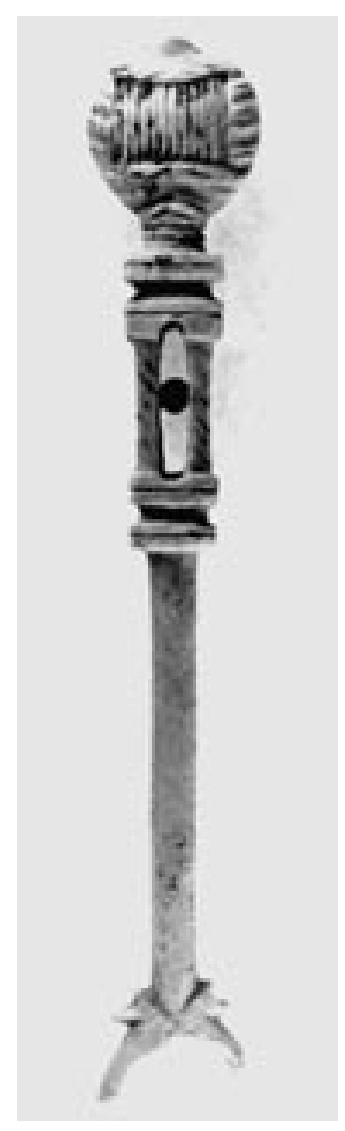

2 Ephesos, Hanghaus 2. Prunkrocken mit Büste KatNr. 2

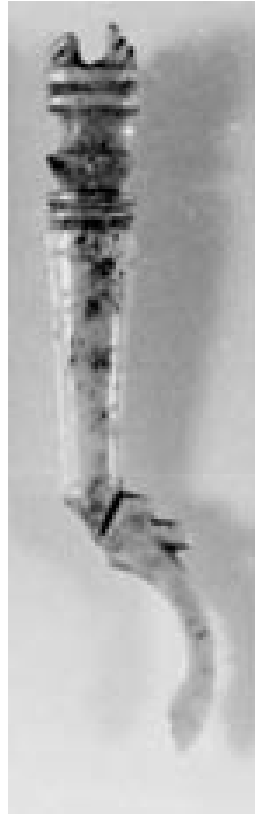

3 Ephesos, Hanghaus 2. Prunkrocken KatNr. 3

römischen Häusern zu unterstreichen ${ }^{4}$. Deswegen jedoch Funde und Fundumstände völlig auszuklammern und sich lediglich in - m. E. falscher - positivistischer Manier auf die Interpretation der landläufig als Baubefund bezeichneten architektonischen Anlagen zu beschränken, scheint mir eine eklatante Geringschätzung oder sogar Mißachtung des archäologischen Quellenmaterials zu sein. Vielmehr ist mit J. Berry die Existenz der Fundgegenstände (»artefactual evidence «) als primäre Quelle für die Aktion und Interaktion der Hausbewohner ${ }^{5}$ zu verstehen; daß diese Prozesse an unterschiedlichen, jeweils geeigneten Stellen eines großen Hauses stattfanden und dadurch der umgebenden Architektur ihren Stempel aufprägten, versteht sich von selbst. Für auszuübende Interaktion mußte jedoch schon bei der Errichtung der Räumlichkeiten eine entsprechende Basis geschaffen werden ${ }^{6}$. Dies macht deutlich, daß eine nur in eine Richtung aufgebaute Beweisführung kaum das Richtige treffen kann.

\section{Spinnrocken aus Ephesos}

Ephesos, Statthaltersitz und gewissermaßen Hauptstadt der Provinz Asia spätestens ab 29 v. Chr., besticht nicht nur durch sein großflächig ausgegrabenes Stadtgebiet, sondern auch durch die Qualität der Befunde und Funde, besonders in den ausgedehnten Privathäusern am Nordabhang

\footnotetext{
4 Dickmann, Domus 281 ff. 375 ff.: »Bereich der domus als Ort vielfältiger Begegnungen zwischen Bewohnern und Besuchern in erster Linie als sozial distinktiven Raum«.

5 J. Berry, Household Artefacts: Re-interpreting Roman Domestic Space, 22. Suppl. JRA (1997) 183-195.

6 S. P. Ellis, Roman Housing (2000) 36.
} 
des Bülbüldağ. Deren Erforschung wurde gerade im letzten Jahrzehnt besonders intensiviert, wodurch viele neue Erkenntnisse erarbeitet werden konnten ${ }^{7}$. Diese erlauben nun auch einen besseren Einblick in die Bau- und Zerstörungsgeschichte und die Befundsituation des Hanghauses 2. Die im Folgenden zu besprechenden Spinnrocken stellen eine sehr kleine Gruppe innerhalb des umfangreichen und variantenreichen Fundmaterials dar'.

Bezüglich der Artefakte für die Textilbearbeitung bzw. -erzeugung läßt sich für das Hanghaus 2 in Ephesos zusammenfassen: Weben und Nähen sind nahezu ausschließlich durch isoliert vorhandene Werkzeuge belegt, vor allem durch Webgewichte aus gebranntem Ton und Nähnadeln aus Bein. Auch von der funktionalen Werkzeuggruppe für das Spinnen (Spindel, Spinnwirtel, Rocken $)^{9}$ sind nur vereinzelte Exemplare erhalten. Die meist aus organischem Material hergestellten Spindeln fehlen im Fundspektrum beider Hanghäuser sogar völlig, lediglich ein einziger Spindelhaken aus Bronze ist im Hanghaus 2 belegt $^{10}$; Spinnwirtel sind selten. Ebenso wie die Spindeln fehlen auch funktionstüchtige Spinnrocken unter den Funden. Die Gestalt der in diesem Rahmen besprochenen Fundgegenstände ist zwar eindeutig auf Spinnrocken zurückzuführen, etliche Details schließen eine Benutzung im Arbeitsprozeß jedoch aus.

\section{Spinnrocken aus dem Hanghaus 2}

KatNr. 1: Venuskunkel (Abb. 1a. b)

Verwahrung: Efes Müzesi, Selçuk (Inv. 16/25/75); derzeit Schauräume.

FO: Hanghaus 2, Wohneinheit 2, SR 27; über Boden, K 3/70; 15. 9. 1970.

Kontext/Mitfunde: Faltenflasche ${ }^{11}$, Standartenbekrönung, Alabastervase, Gefäßkeramik ${ }^{12}$. Die Zerstörung des Hofes gibt mit etwa $270 \mathrm{n}$. Chr. einen terminus ante quem für die Entstehung der Fingerkunkel ${ }^{13}$.

Erhaltungszustand: Bis auf einen kleinen abgebrochenen Teil vom äußersten Ende der Palmette auf der Ringöse und Fehlstellen am Rücken und Hinterkopf der Statuette vollständig erhalten, in zwei Teile gebrochen.

Material: Bein.

Maße: $\mathrm{L}=22,8 \mathrm{~cm}$.

Publikation: E. Trinkl, »Die spinnen, die Römerinnen«, in: L. Dollhofer u. a. (Hrsg.), Altmodische Archäologie. Festschrift F. Brein, Forum Archaeologiae 14/III/2000 (http://farch.net) Abb. 5; E. Trinkl, Bemerkungen zu kleinasiatischen Venuskunkeln, Bulletin Instrumentum 15, 2002, 31-35 Abb. 1.
Vergleichsliteratur: König, Fingerkunkel; Cremer, Venuskunkeln; Bíró, Goddess.

Ovaler, glatter Schaft. Die glatte, undekorierte, abgeflachte Öse setzt mit stilisierten Blättern am Schaft an, am untersten Ende der Ringöse befindet sich eine siebenblättrige Palmette. Am anderen Ende des Stabes steht die Statuette einer Venus im 'Pudica-Typus' mit stilisierter Delphinstütze, die sich im Bereich des rechten unteren Oberschenkels anlehnt.

Die Statuette ist sauber gearbeitet: gelängte, frontale Figur mit langem Hals und relativ kleinem Kopf, Haar mit Mittelscheitel zur Seite gestrichen, Schulterlocken, gezacktes Diadem. Das rechte Bein ist das Standbein, das linke wenig gebeugt. Aphrodite hält den rechten Unterarm vor bzw. unter die Brust, den herabgleitenden Mantel faßt sie mit der linken Hand vor der Scham. Dabei entstehen zwei um die Hüften führende, horizontale Falten in der Form kleiner Wülste; der geraffte Mantel fällt in einer breiten, glatten Falte vor den Beinen zu Boden. Die horizontalen Wülste laufen auf der Rückseite um, der restliche Mantel liegt in schrägen Falten. Rücken und Kopf sind an der Rückseite abgesprungen.

7 Krinzinger, Baugeschichte, passim; Thür, Hanghaus; Lang-Auinger, Hanghaus, passim; E. Rathmayr in: Krinzinger, Wohneinheiten.

8 Allgemein zu den Befunden und Funden des Hanghauses 2 s. Ladstätter, Chronologie, bes. 14 ff. - Die Spinnrokken sind nur ein Teil jener Artefakte für die Textilverarbeitung, deren Aufarbeitung in der Hand der Verf. liegt. Zu den Funden für die Textilverarbeitung aus dem Hanghaus $1 \mathrm{~s}$. Trink1, Artefakte $313 \mathrm{ff}$.

9 Catull. 64; E. Wayland Barber, Women's Work: The First 20000 Years. Women, Cloth, and Society in Early Times (1995); K. von Kurzynski, »... und ihre Hosen nennen sie bracas«. Textilfunde und Textiltechnologie der Hallstattund Latènezeit und ihr Kontext, Internationale Archäologie 22 (1996) 7 ff.

10 Eine Spindeltülle aus Bronze, wie sie vielfach in Sardes belegt ist, konnte bisher im Fundmaterial des Hanghauses 2 nur ein einziges Mal identifiziert werden; S. Jilek in: Thür, Hanghaus 394 Kat. B67.

11 M. Schätzschock wird diese Flasche im Zusammenhang mit den Glasfunden aus den Wohneinheiten 1 und 2 besprechen.

12 Nach der freundlichen Auskunft von S. Ladstätter stammt die Gefäßkeramik aus dem 3. Viertel des 3. Jhs.

13 H. Vetters, AnzWien 108, 1971, 100 f. Taf. 22 (Standarte). Ladstätter, Chronologie 16 ff. 24 ff.; E. Rathmayr in: Krinzinger, Wohneinheiten. 
Als Träger der Statuette fungiert eine schmale, dreigeteilte Basis. In ihrer Mitte befindet sich eine tief eingeschnittene Kehle, oben und unten liegt je eine Leiste, die durch eine schmale Horizontalrille akzentuiert ist.

KatNr. 2: Fingerkunkel mit Büste und tetrastylem Aufsatz mit Kügelchen (Abb. 2)

Verwahrung: verschollen.

FO: Hanghaus 2, Wohneinheit 2, SR 27a; Schutt, K 6/69, Fundnr. 74/69; 1969.

Kontext/Mitfunde: Gefäßkeramik ${ }^{14}$.

Erhaltungszustand: Beide Enden (Kopf der Büste, Ringöse z. T.) verbrochen, sonst vollständig erhalten.

Material: Bein.

Maße: erh. $\mathrm{L}=15,4 \mathrm{~cm}$.

Publikation: unpubliziert.

Vergleichsliteratur: zu tetrastylem Aufsatz mit Kugel in der Mitte bei einer Bronzenadel vgl. F. Petrie, Objects of Daily Use, British School of Archaeology in Egypt (1927) 24 Taf. 19, 74; bei Henkeln(?): Waldbaum, Sardis Nr. 941; bei anderen Beingeräten s. T. M. Bíró, Bonecarvings from Brigetio in the collection of the Hungarian National Museum, ActaArchHung 39, 1987, 171 Nr. 55 Abb. 11; S. Jilek in: Thür, Hanghaus 393 f. B25 Taf. 236. 240. Zur Büste ${ }^{15}$ vgl. M. Feugère, Les spatules à cire à manche figuré, in: C. M. Hüssen (Hrsg.), Provinzialrömische Forschungen. Festschrift G. Ulbert (1995) 328 Nr. 19 Abb. 6c.

An ovalem(?) Schaft mit stilisierten Blättern teilweise erhaltene Grifföse. Am oberen Ende des glatten Schaftes folgt ein tetrastyler Aufsatz mit mehrfach profilierten Abschlüssen; innerhalb des geschlitzten Abschnitts befindet sich eine frei bewegliche Kugel, die 'Säulchen' sind mit schrägen Kerben verziert. Darüber eine stark stilisierte Büste ohne Kopf. Auf der Vorderseite der Büste etwa rechteckiges, senkrecht gestreiftes Feld mit eingezogener oberer und unterer Langseite, das Gewand ist durch annähernd horizontale Rillen angegeben.

KatNr. 3: Rocken mit Ansatz eines tetrastylen Aufsatzes (Abb. 3)

Verwahrung: Depot des österreichischen Grabungshauses in Selçuk.
FO: Hanghaus 2, Wohneinheit 2, SR 27c; aus dem Kanal, K 7/72, Fundnr. 72/110; 2. 10. 1972.

Kontext/Mitfunde: Fünf Beinnadeln und eine Nähnadel; Glasfragmente $^{16}$; Gefäßkeramik ${ }^{17}$.

Erhaltungszustand: Je ein Teil des Schaftes und der Ringöse erhalten.

Material: Bein.

Maße: erh. $\mathrm{L}=8,4 \mathrm{~cm}$.

Publikation: unpubliziert.

Vergleichsliteratur: zu tetrastylem Abschnitt s. KatNr. 2.

Die Ringöse setzt mit zwei stilisierten Blättern und dekorierter Zwickelfläche an den sich kontinuierlich verbreiternden, glatten Schaft an, der verhältnismäßig kurz ist. Zwischen je einem doppelt profilierten Abschnitt liegt ein konvex-konkaves Zwischenstück; darüber Ansatz eines geschlitzten, mit Spiralen dekorierten Abschnitts (tetrastyler Aufsatz).

KatNr. 4: Venuskunkel (Abb. 4a. b)

Verwahrung: Efes Müzesi, Selçuk (Inv. 137/60/78).

FO: Hanghaus 2, Wohneinheit 6, R 31 (H2/78/15); Fundnr. 201/78; 30. 8. 1978.

Kontext/Mitfunde: Füllschutt R 31: Material in Sturzlage aus dem Nordbereich der Wohneinheit 4 (R 6 oder R 7 bzw. aus dem ersten Stockwerk in diesem Bereich) ${ }^{18}$.

Erhaltungszustand: Beide Enden verbrochen, sonst vollständig.

Material: Bein.

Maße: erh. $\mathrm{L}=12,9 \mathrm{~cm}, \max . \mathrm{B}=1,4 \mathrm{~cm}$.

Publikation: unpubliziert.

Vergleichsliteratur: König, Fingerkunkel; Cremer, Venuskunkeln; Bíró, Goddess.

Der relativ kurze, glatte Stab mit ovalem Querschnitt endet an einer Seite mit einer nur zu einem kleinen Teil erhaltenen Ringöse; der Ansatz ist mit zwei kleinen Fortsätzen und einer V-förmigen Kerbe dekoriert. Am anderen Ende steht auf einer doppelt profilierten Basis mit tiefer Kehle, deren unterster Streifen mit Kerbschnitt dekoriert ist, eine Frauengestalt im 'Venus-Pudica-Typus'; der Kopf fehlt. Oberkörper, Arme und Finger sind stark gelängt. Die Statuette ist relativ flach gearbeitet; dies dürfte die Folge eines flachen Rohlings sein, wie auch an der Rückseite zu beobachten, wo am Rücken der Statuette der Kanal des Knochens bereits ansetzt ${ }^{19}$.

14 Nach der freundlichen Auskunft von S. Ladstätter stammt die Gefäßkeramik aus dem 2. Viertel des 3. Jhs.

15 Die Gestaltung der Brust der Büste erinnert an bronzene Athenabüsten, die als Griffe für Wachsspachteln Verwendung finden; M. Feugère, Les spatules à cire à manche figuré, in: C. M. Hüssen (Hrsg.), Provinzialrömische Forschungen. Festschrift G. Ulbert (1995) 328 Nr. 19 Abb. 6c. Die kleinformatige Athena an einer Basilika in Leptis Magna trägt eine ähnlich wenig strukturierte, annähernd rechteckige Ägis mit eingezogenen Seiten; LIMC II 2 (1984) 1101 s. v. Athena/Minerva Nr. 377 (F. Canciani).

16 M. Schätzschock wird die Bruchstücke im Zusammenhang mit den Glasfunden aus den Wohneinheiten 1 und 2 besprechen.

17 Nach der freundlichen Auskunft von S. Ladstätter stammt die Gefäßkeramik aus dem 3. Viertel des 3. Jhs.

${ }_{18}$ H. Thür, Die Bauphasen der Wohneinheiten 4 (und 6), in: Krinzinger, Baugeschichte 58; dies. in: Thür, Hanghaus $101 \mathrm{f}$. Die in Wohneinheit 4 festgestellten Bauarbeiten in der letzten Benutzungsphase IV' machen eigentlich eine gleichzeitige Einlagerung kostbarer Gegenstände wenig wahrscheinlich; dies. in: Krinzinger, Baugeschichte 59. 64. Zur Datierung von Wohneinheit 6, R 31, sog. Marmorsaal, vgl. K. Koller, Die Pilasterkapitelle aus dem 'Marmorsaal' der Wohneinheit 6, in: Krinzinger, Baugeschichte, bes. $135 \mathrm{f}$.

19 Der freiliegende Knochenkanal auf dem Rücken der Venus macht deutlich, daß dieser Gegenstand nicht auf Allansichtigkeit gearbeitet ist, welche allerdings bei der Verwendung eines Spinnrockens unvermeidlich ist. 

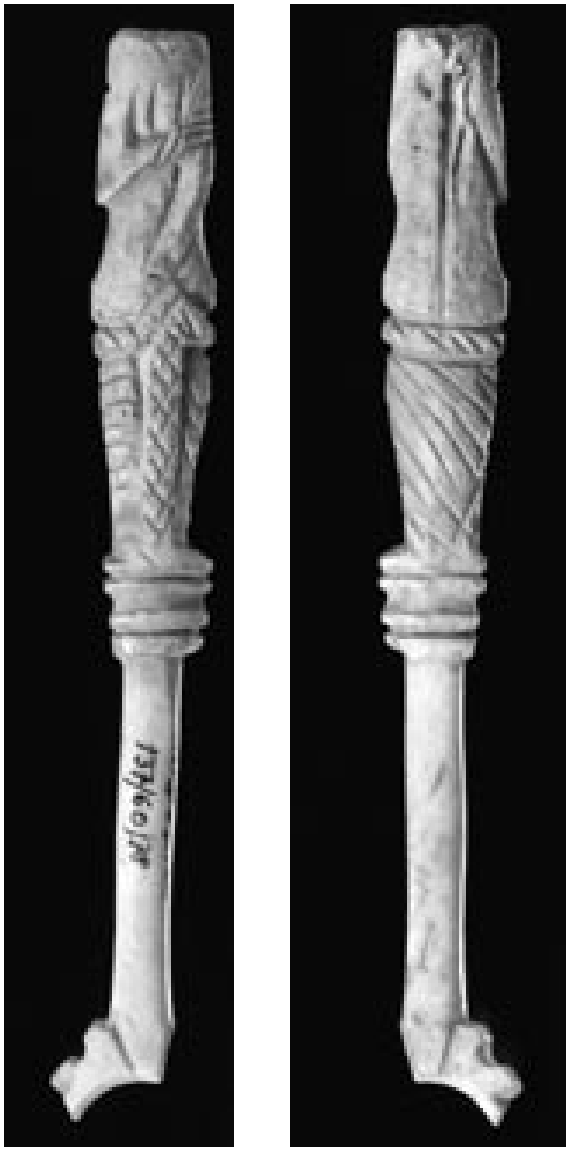

4a-b Ephesos, Hanghaus 2. Venuskunkel KatNr. 4, Efes Müzesi Selçuk (Inv. 137/60/78)

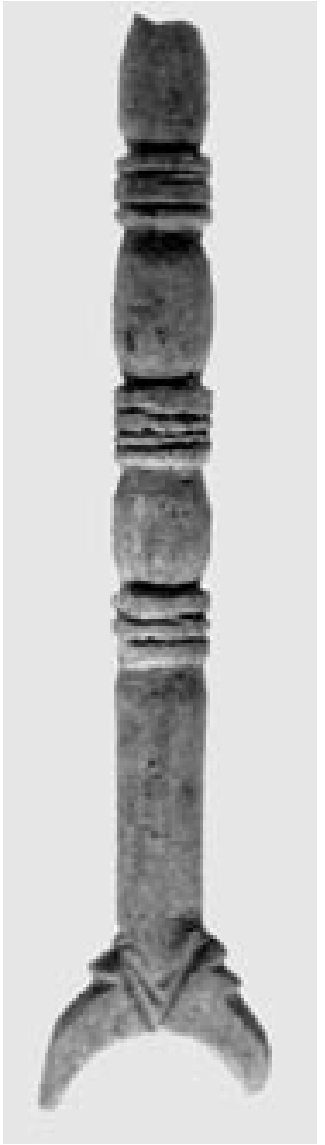

5 Ephesos, Hanghaus 2. Prunkrocken KatNr. 5

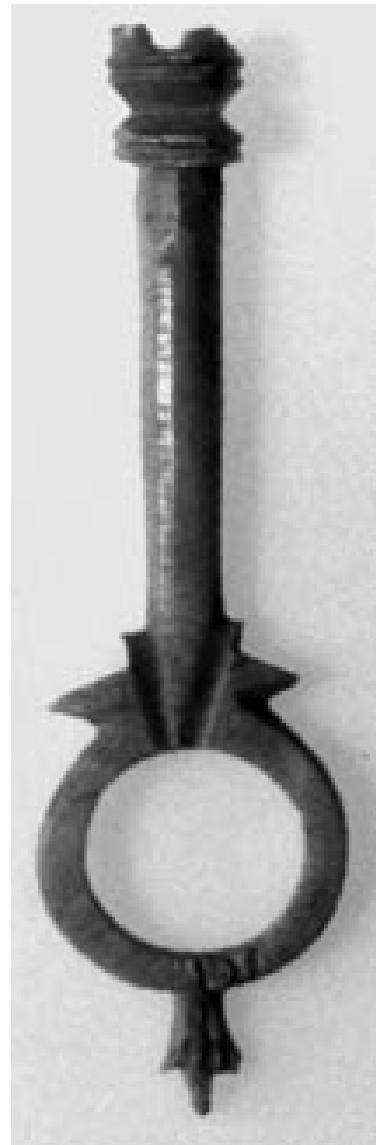

6 Ephesos, Hanghaus 2. Venuskunkel KatNr. 6 , Efes Müzesi Selçuk (Inv. 136/59/80)
KatNr. 5: Rockenfragment (Abb. 5)

Verwahrung: verschollen.

FO: Hanghaus 2, Wohneinheit 7, R 38e, nördlich des Nymphäums; Schutt, K 19/81 (Fundnr. 86/81), H2/81/ 488; 16. 9. 1981.

Kontext/Mitfunde: Ziegelfragment mit Bienenstempel (Fundnr. 85/81), drei Beinnadeln.

Erhaltungszustand: Beide Enden verbrochen, aber Schaft gut erhalten.

Material: Bein.

Maße: erh. $\mathrm{L}=10,8 \mathrm{~cm}$.

Publikation: unpubliziert.

Vergleichsliteratur: E. Deschler-Erb, Römische Beinartefakte aus Augusta Raurica, Forschungen in Augst 27/1 (1998) 192 Nr. 4522-4523; M. T. Bíró, Catalogi Musei Nationalis Hungarici II (1994) Nr. 456 Taf. 191.

Die etwa zur Hälfte erhaltene, flache Ringöse setzt mit kurzen spitzen Fortsätzen am vorerst glatten Schaft an, die Zwickelfläche ist mit einer doppelten keilförmigen Ritzung dekoriert. Über dem glatten Schaftabschnitt sind drei perlenartige Verdickungen erhalten, die durch glatte, doppelt eingeschnittene Abschnitte voneinander getrennt sind.
KatNr. 6: Rockenfragment (Abb. 6)

Verwahrung: Efes Müzesi, Selçuk (Inv. 136/59/80).

FO: Hanghaus 2, Wohneinheit 7, R 38a; Fundnr. H2/80/ 65; 10. 9. 1980.

Kontext/Mitfunde: aus Schutt.

Erhaltungszustand: Ringöse und glatter Schaft vollständig erhalten, oberer Abschluß abgebrochen.

Material: Bein.

Maße: erh. $\mathrm{L}=8,6 \mathrm{~cm}$.

Publikation: unpubliziert.

Vergleichsliteratur: zu tetrastylem Abschnitt s. KatNr. 2. Wenig abgeflachter Schaft mit rundem Querschnitt schließt auf der einen Seite mit abgeflachter Ringöse ab, am Übergang liegen besonders spitz ausgeführte stilisierte 'Blätter'. Ein breites V dekoriert den Übergang von Öse und Schaft. Am äußersten Ende der Ringöse liegt eine schlanke, stark stilisierte Palmette. Am oberen Ende des Schaftes schließt ein in der Mitte tief eingeschnürter, horizontal profilierter Abschnitt mit viereckigem Querschnitt an; darüber, allerdings großenteils verbrochen, Ansatz eines in der Mitte ausgehöhlten, vermutlich tetrastylen Abschnitts. Die Oberfläche ist nicht geglättet, teilweise blieben wenig kantige Grate stehen. 
KatNr. 7: Rockenfragment (Abb. 7)

Verwahrung: Depot des österreichischen Grabungshauses in Selçuk.

FO: Hanghaus 2, Wohneinheit 2 (Raumzuordnung nicht gesichert); Fundnr. c, K 1/69; 1969.

Kontext/Mitfunde: aus Schutt.

Erhaltungszustand: Ringöse vollständig, kleiner Abschnitt des Schaftes erhalten.

Material: Bein

Maße: erh. $\mathrm{L}=5,45 \mathrm{~cm}$.

Publikation: unpubliziert.

Vergleichsliteratur: zum Typus s. König, Fingerkunkel. Ringöse mit dreiblättrigem, kurzem Fortsatz am äußersten Ende der Ringöse und mit je zwei abgerundeten Fortsätzen an beiden Seiten des Schaftansatzes. Zwei Rillen im untersten Abschnitt des runden Schaftes.

KatNr. 8: Rockenfragment (Abb. 8)

Verwahrung: verschollen.

FO: Hanghaus 2, Wohneinheit 4, K 2/63; Fundnr. 9; 24. 8. 1963.

Kontext/Mitfunde: Verschüttung Wohneinheit $4^{20}$; Flügelfragment, weibliches Köpfchen im Typus Aphrodite Anadyomene, Becken aus Marmor, Säulchen aus Marmor, Reibstein ${ }^{21}$.
Erhaltungszustand: Oberer Teil und Schaft fehlen teilweise.

Material: Bein.

Maße: erh. $\mathrm{L}=7,7 \mathrm{~cm}$.

Publikation: unpubliziert.

Vergleichsliteratur: zum Typus s. König, Fingerkunkel.

Mit stilisierten Blättern setzt die Öse an den Schaft an, am äußersten Ende der Öse büschelige Verzierung.

KatNr. 9: Rockenfragment (Abb. 9)

Verwahrung: Depot des österreichischen Grabungshauses in Selçuk.

FO: unstratifiziert; mit großer Wahrscheinlichkeit aus einem der beiden Hanghäuser.

Kontext/Mitfunde: unstratifiziert.

Erhaltungszustand: Ringöse teilweise und oberes Ende verbrochen.

Material: Bein.

Maße: erh. $\mathrm{L}=7 \mathrm{~cm}$.

Publikation: unpubliziert.

Vergleichsliteratur: zum Typus s. König, Fingerkunkel.

Glatter Schaft mit ovalem Querschnitt. Je zwei blattartige

Fortsätze beiderseits des Ansatzes der Ringöse, X-Ritzung am verbreiterten Ansatz der Ringöse. Reste eines profilierten Übergangs am obersten erhaltenen Ende des Schaftes.

\section{Spinnrocken von anderen Fundorten in Ephesos bzw. aus der Umgebung von Ephesos}

KatNr. 10: Rockenfragment (Abb. 10)

Verwahrung: Efes Müzesi, Selçuk (Inv. 172/51/91).

FO: Damianosstoa, Sarkophag.

Kontext/Mitfunde: Frauengrab, Anfang 3. Jh. n. Chr.: Spindel, Spinnwirtel; weitere Grabbeigaben: Goldschmuck, Glasarmreif, Beinnadeln, Bronzespiegel, Nadelbüchse aus Bronze, Glasunguentarien.

Erhaltungszustand: Ringöse teilweise und oberes Ende verbrochen; Oberfläche stark angegriffen.

Material: Bein.

Maße: erh. $\mathrm{L}=14,3 \mathrm{~cm}$.

Publikation: Trinkl, Set 80 Abb. 1; E. Trink1, Die Kleinfunde aus Sarkophag und Grabhaus an der Ostseite des Panayırdağ (Grabung 1991), in: D. Knibbe - H. Thür (Hrsg.), Via Sacra Ephesiaca II, BerMatÖAI 6 (1995) 69. 75 Nr. 12 Taf. 16.

Vergleichsliteratur: Waldbaum, Sardis Nr. 940 Taf. 53; zum Typus s. König, Fingerkunkel.

An die abgeflachte Ringöse schließt ein kurzer glatter Schaft an. Darüber folgen fünf dekorierte Abschnitte; die unteren drei sind durch schräg geführte Ritzungen dekoriert, darüber folgen zwei tief gekehlte Abschnitte. Der oberste erhaltene Teil ist als Pinienzapfen mit gekreuzter, flacher Ritzung gestaltet. Am Ansatz darüber ist der Rocken gebrochen.

KatNr. 11: Rockenfragment (Abb. 11)

Verwahrung: Depot des österreichischen Grabungshauses in Selçuk.
FO: Staatsmarkt, Basilika Fundnr. 9230.

Kontext/Mitfunde: unbestimmt.

Erhaltungszustand: Teil der Grifföse und oberer Abschluß fehlen.

Material: Bein.

Maße: erh. $\mathrm{L}=14,1 \mathrm{~cm}$.

Publikation: unpubliziert.

Vergleichsliteratur: Einfache Kunkel ohne stilisierte Blätter am Schaftansatz vgl. M. Mackensen, Das römische Gräberfeld auf der Keckwiese in Kempten, Cambodunumforschungen 4 (1978) 228 Grab 102 Nr. 8; M. Bonghi (Hrsg.), Richerche a Pompei. L'insula 5 della Regio VI dalle origini al 79 d.C. (1984) Taf. 182, 17; 183, 1.

An die nur teilweise erhaltene Grifföse schließt ohne Verzierungen ein glatter, mit schräger Ritzung verzierter Schaft an. Darüber folgt zwischen je einer tief eingeschnittenen Kehle eine Spule mit gekreuzter Ritzung. Oberhalb einer doppelt horizontal gekerbten, sich etwas verjüngenden Basis ist der Rocken gebrochen.

KatNr. 12: Rockenfragment (Abb. 12)

Verwahrung: Depot des österreichischen Grabungshauses in Selçuk.

FO: Staatsmarkt, Basilika Fundnr. 60/19.

Kontext/Mitfunde: unbestimmt.

Erhaltungszustand: Nur unterster Abschnitt der Ringöse erhalten.

Material: Bein.

Maße: erh. $\mathrm{L}=2,6 \mathrm{~cm}$

${ }^{20}$ H. Thür in: Thür, Hanghaus $101 \mathrm{f}$.

${ }^{21}$ Fundjournal (im Archiv des Instituts für Kulturgeschichte der Antike, ÖAW) 1963, S. 36. 


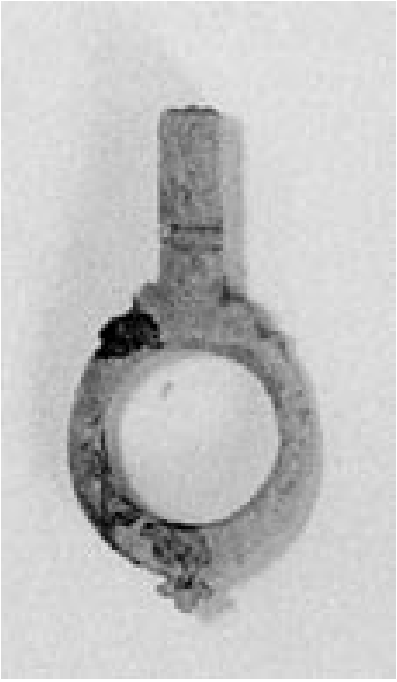

7 Ephesos, Hanghaus 2. Prunkrocken KatNr. 7

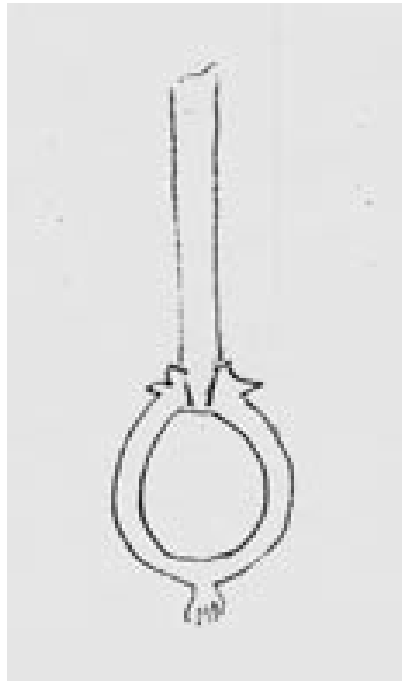

8 Ephesos, Hanghaus 2. Prunkrocken KatNr. 8 (Skizze Fundjournal)

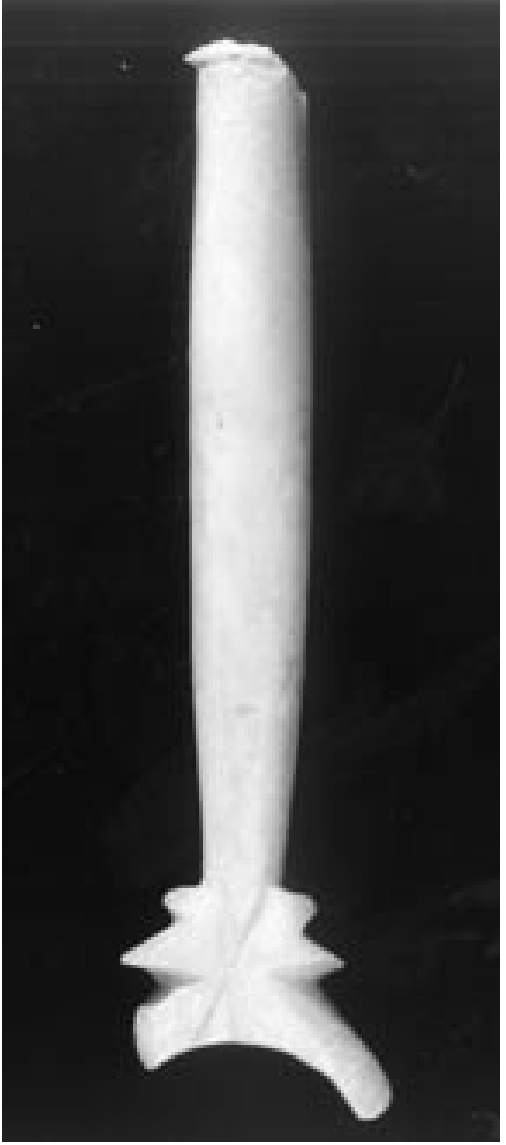

9 Ephesos. Prunkrocken KatNr. 9
Publikation: unpubliziert.

Vergleichsliteratur: zum deltaförmigen Fortsatz s. KatNr. 10.

Der unterste Teil der abgeflachten Ringöse trägt einen dekorativen deltaförmigen Fortsatz. Die Oberfläche zeigt deutliche Kratzer.

KatNr. 13: Rockenfragment (Abb. 13)

Verwahrung: Depot des österreichischen Grabungshauses in Selçuk.

FO: Staatsmarkt, Basilika, o. Fundnr.

Kontext/Mitfunde: unbestimmt.

Erhaltungszustand: Ringöse teilweise und oberes Ende verbrochen.

Material: Bein

Maße: erh. $\mathrm{L}=7,45 \mathrm{~cm}$.

Publikation: unpubliziert.

Vergleichsliteratur: zum Typus s. König, Fingerkunkel. Die Ringöse setzt mit je zwei zugespitzten Fortsätzen an den glatten Schaft an. Darüber folgt ein vierfach horizontal eingekerbter Abschnitt, der eine Überleitung zu dem darüberliegenden, leider verlorenen dekorativen Abschnitt bildet.

KatNr. 14: Rockenfragment (Abb. 14)

Verwahrung: unbekannt, keine Autopsie.

FO: Magnesisches Tor, Abwasserkanal, K 4/79.

Kontext/Mitfunde: Münze Constantius II.
Erhaltungszustand: Vollständige Ringöse und darüber folgender Schaft.

Material: Bein.

Publikation: unpubliziert.

Vergleichsliteratur: zu Palmette s. KatNr. 6.

Die abgeflachte Ringöse schließt mit je zwei spitzen Fortsätzen auf beiden Seiten an den glatten Schaft an, die Zwickelfläche ist mit einer X-förmigen Ritzung verziert. Am äußersten Punkt der Ringöse sitzt eine stilisierte Palmette mit drei Blättern. Über dem glatten Schaft folgt eine zwischen vier an der Unterseite und zwei an der Oberseite horizontalen Bändern liegende, etwas gelängte Perle, die auf Grund ihrer gekreuzten Ritzung als stilisierter Pinienzapfen angesprochen werden kann.

KatNr. 15: Rockenfragment (Abb. 15)

Verwahrung: Efes Müzesi, Selçuk (Inv. 5/25/73).

FO: Ankauf Efes Müzesi.

Kontext/Mitfunde: keine Angaben; auf Grund des Erhaltungszustands vermutlich aus sepulkralem Kontext.

Erhaltungszustand: Oberfläche stark angegriffen, Grifföse fehlt teilweise.

Material: Bein.

Maße: erh. $\mathrm{L}=17,0 \mathrm{~cm}$.

Publikation: unpubliziert.

Vergleichsliteratur: zum tetrastylen Abschnitt s. KatNr. 2.; zum Pinienzapfen s. KatNr. 10.

Die abgeflachte Ringöse schließt mit je zwei spitzen 


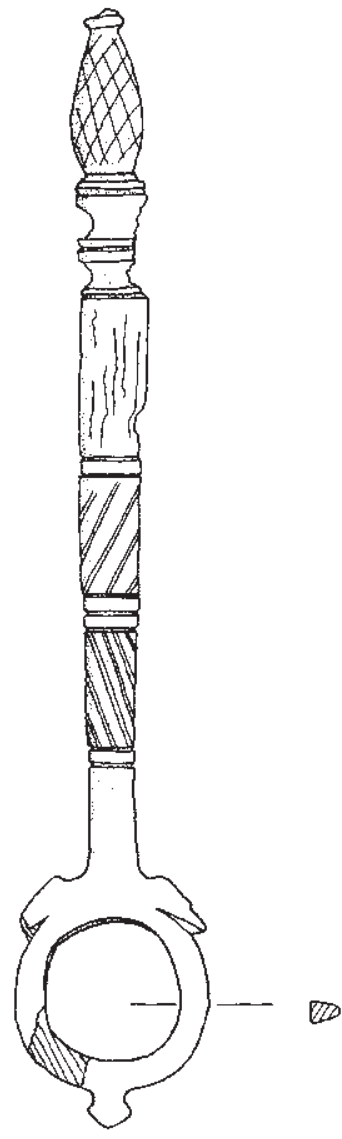

10 Ephesos, Damianosstoa. Prunkrocken KatNr. 10
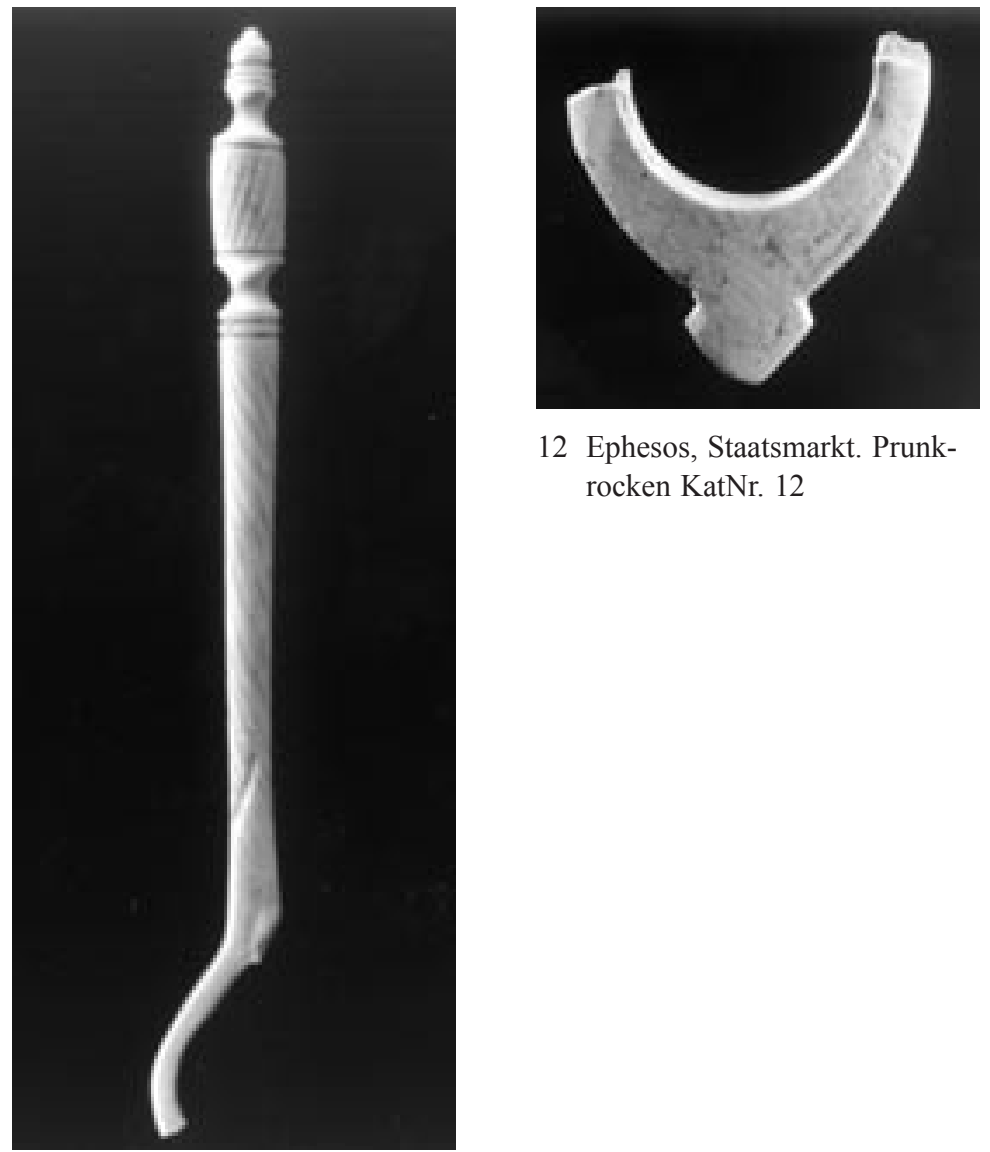

12 Ephesos, Staatsmarkt. Prunkrocken KatNr. 12
Fortsätzen auf beiden Seiten an den kurzen, glatten Schaft an, die Zwickelfläche ist mit einer V-förmigen Ritzung verziert. Oberhalb des Schaftes liegen zwei tetrastyle Ädikulen übereinander, die Säulchen sind mit schrägen Ritzungen verziert. Ein mehrfach horizontal geritztes Zwischenglied mit einer tiefen Kehle leitet zu einem bekrönenden, mit gekreuzter flacher Ritzung verzierten Pinienzapfen über.

\section{Gemeinsamkeiten der hier vorgestellten Artefakte}

Alle an dieser Stelle vorgestellten Exemplare sind aus Bein gefertigt ${ }^{22}$. Eine weitere Gemeinsamkeit ist der aus mehreren unterschiedlich gestalteten Abschnitten bestehende Aufbau. Eine runde Öse am unteren Ende ermöglicht die Benennung der Artefakte als Spinnrocken bzw. Fingerkun-

22 Zahlreiche Spinnrocken sind auch aus anderen Materialien belegt, beispielsweise Glas, Gagat, Elfenbein, Bernstein. Bei ihnen handelt es sich ebenso wie bei den vorgestellten ephesischen Exemplaren um Gegenstände, deren Verwendung als Werkzeug in diesem Fall auf Grund der Materialwahl zu bezweifeln ist. R. Gottschalk, Ein spätrömischer Spinnrocken aus Elfenbein, AKorrB1 26, 1996, 483-500; Out of Rome. Augusta Raurica/Aquincum (Ausstellungskat. 1997) 315 Abb. 311: Bronzespindel, Glasrocken mit Vogel aus einem Frauengrab südlich von Aquin-

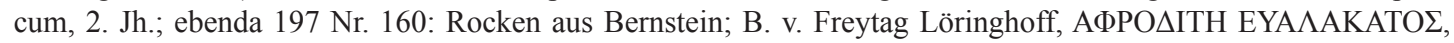
in: M. O. Jentel - G. Deschênes-Wagner (Hrsg.), Tranquillitas. Mélanges en l'honneur de Tram tan Tinh (1994) 191-198: Venuskunkel aus Elfenbein unbekannter Herkunft. - In diesem Zusammenhang sollten auch die meist als 'stiring-rods' bezeichneten, gedrehten Glasstäbe erwähnt werden, bei denen ein Ende als Ring gestaltet ist. Es könnte sich auch in diesen Fällen um Rocken handeln; vgl. Trinkl, Set; C. S. Lightfoot - M. Arslan, Ancient Glass of Asia Minor: the Yüksel Erimtan Collection (1992) Nr. 147-149; S. Sakl-Oberthaler - K. Tarcsak, Römerzeitliche Glasobjekte aus Wien, FOWien 5, 2002, 148 f. Abb. 9. 


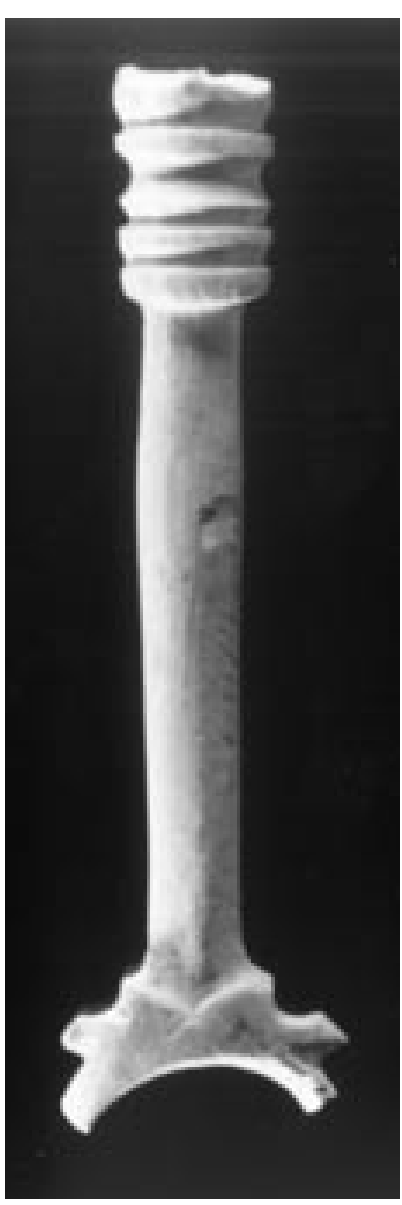

13 Ephesos, Staatsmarkt. Prunkrocken KatNr. 13

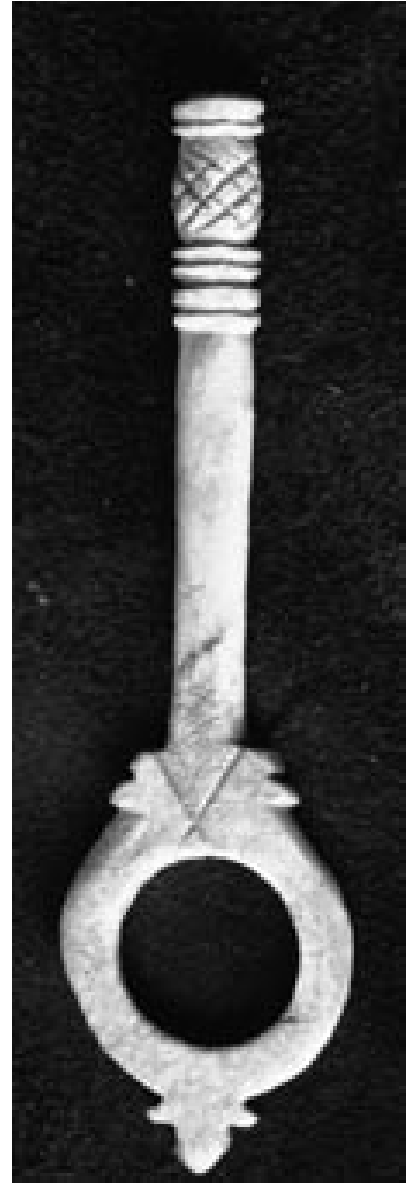

14 Ephesos, Magnesisches Tor. Prunkrocken KatNr. 14

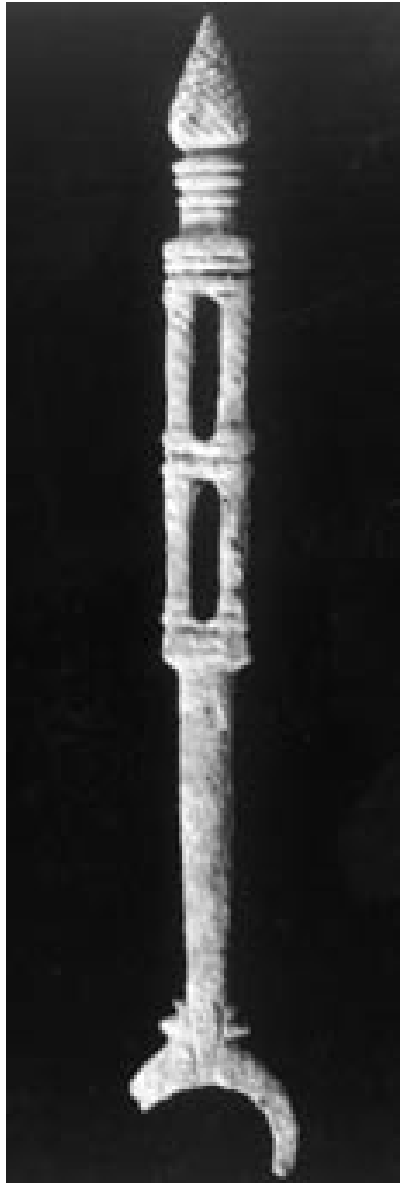

15 Rockenfragment KatNr. 15, Efes Müzesi Selçuk (Inv. 5/25/73)

$\mathrm{ke}^{23}$. Die im Querschnitt abgeflachten Ringösen tragen an ihrem äußersten Ende eine Verzierung, die in Form einer Palmette, eines einfachen Büschels oder eines Deltas gestaltet is $\mathrm{t}^{24}$. Den meisten Spinnrocken gemeinsam ist auch der verspielt gestaltete Übergang von Ringöse zu Schaftansatz, lediglich bei KatNr. 11 bleibt diese Passage undekoriert ${ }^{25}$. Bei den seitlich an der Verbin-

23 König, Fingerkunkel.

24 Es darf angenommen werden, daß es sich bei den einfachen Fortsätzen um stilisierte Palmetten handelt. Bei einem fragmentierten Exemplar aus Korinth sind die Palmette und die Ringöse ineinander integriert; Davidson, Corinth 280 Nr. 2389 Taf. 119. Bei spätantiken Exemplaren wird dieses Element zunehmend abstrahiert; Bíró, Goddess. - Bei einem Exemplar aus Bronze, das sich heute in Privatbesitz befindet, sitzt auf einem Ende ein Vogel, das andere endet wie die ephesischen Fingerkunkeln aus Bein als Ringöse, an deren äußerstem Ende ein kleiner Zapfen angebracht ist; Cremer, Spinnrocken 242 f. Taf. 25, 2; Cremer, Fingerkunkel 328 f. Taf. 29, 3; s. auch Waldbaum, Sardis Nr. 940. Ähnliche Zapfen sind auch bei Exemplaren aus Bein und Glas zu beobachten; König, Fingerkunkel 129 Abb. 1.

25 Vergleichbare Spinnrocken aus Gräbern werden bei H. Mikler diskutiert; H. Mikler, Die römischen Funde aus Bein im Landesmuseum Mainz, Instrumentum Monographie 1 (1997) 53 f. Abb. 6 Taf. 39, 8 f.; s. auch Cremer, Venuskunkeln Abb. 5. - Glatte Nadeln mit Ringösen, die weniger reich verzierten Fingerkunkeln nahestehen, könnten, wie von K. Gostenčnik vorgeschlagen, als Haarnadeln verwendet worden sein: K. Gostenčnik, Die Kleinfunde aus Bein vom Magdalensberg, Carinthia I 186, 1996, bes. 117 Taf. 4, 1. Die von ihr festgestellte Farbe ist jedoch ebenso an einem funktionsentleerten, reich dekorierten Rocken vorstellbar. Vgl. dies., Pre- and Early Roman Bone and Antlar Manufacturing in Kärnten, Austria, in: A. M. Choyke - L. Bartosiewicz (Hrsg.), Crafting Bone: Skeletal Technologies through Time and Space. Proceedings of the 2nd Meeting of the Worked Bone Research Group, BAR International Series 937 (2001) bes. 385 Abb. 5, 5-6. 
dungsstelle von Stab und Öse ansetzenden, oft sehr spitz zulaufenden Enden ist am ehesten an stilisierte Blätter zu denken ${ }^{26}$. Zusätzlich kann der abgeflachte Ansatz des Schaftes mit einer weiteren Verzierung dekoriert sein, einer X- oder V-förmigen Ritzung. An die Ringöse schließt meist ein glatter Stab an, darüber folgen unterschiedlich dekorierte Stababschnitte. Ein Teil des Schaftes kann auch in eine tetrastyle Ädikula ${ }^{27}$ aufgelöst (KatNr. 2. 3. 6. 15) sein.

Auf der der Ringöse gegenüberliegenden Seite, also am oberen Ende des Geräts, befinden sich weitere dekorative Elemente unterschiedlicher Gestalt. Im vorliegenden Fall sind zwei Statuetten (KatNr. 1. 4), eine teilweise erhaltene Büste (KatNr. 2) und zwei Pinienzapfen (KatNr. 10. 15) $)^{28}$ belegt. Bei den übrigen Exemplaren ist dieser Abschnitt nicht erhalten. Auf Grund der Analogien darf aber mit einer dekorativen Gestaltung des oberen Endes des Spinnrockens auch bei den fragmentarisch erhaltenen Exemplaren gerechnet werden.

Von den dekorierten Exemplaren sind vor allem KatNr. 1 und 4 durch die Statuette einer Venus Pudica besonders reich gestaltet; die übrigen Merkmale entsprechen den Charakteristika der Gruppe. Spinnrocken mit Venusstatuetten werden in der Forschung in Nachfolge von G. G. König als Venuskunkeln bezeichnet ${ }^{29}$, dem schließe ich mich in der Folge ebenfalls an.

Für die Datierung der Exemplare aus dem Hanghaus 2 gibt die Zerstörungsgeschichte des Baukomplexes einen terminus ante quem mit einer Erdbebenserie in den 60er Jahren des 3. Jahrhunderts n. Chr. ${ }^{30}$. Aus der ersten Hälfte des 3. Jahrhunderts stammt der Rocken KatNr. 10, der in einem Sarkophag an der sog. Damianosstoa gefunden wurde ${ }^{31}$. Für die anderen Exemplare ist eine zeitliche Einordnung wegen fehlender Kontexte nicht möglich, sie können aber auf Grund der Ähnlichkeiten mit den datierten Exemplaren an diese angeschlossen werden ${ }^{32}$.

Dekorative Elemente der beschriebenen Art sind an einem Werkzeug, das mit der Handhabung von Rohwolle oder Rohgarn zu verbinden ist, nicht vorstellbar ${ }^{33}$. An den feinen Graten und Spitzen würde die Wolle hängen bleiben und das Arbeiten behindern. Darüber hinaus ist das feste Zugreifen an der dafür vorgesehenen Fingerkunkel durch die spitzen Blätter und die am unteren Ende sitzenden Palmetten extrem erschwert ${ }^{34}$. Weiters fällt das proportionale Mißverhältnis zwischen den dekorativ gestalteten Abschnitten und den direkt von der Funktionalität abgeleiteten, glatten Abschnitten der Rocken besonders auf ${ }^{35}$.

26 Ähnliche Fortsätze sind auch an Rocken aus anderem Material zu beobachten: A. Wasowicz, Deux aspects de l'art funéraire dans les antiquités du Bosphore cimmérien, RLouvre 37, 1987, 268 ff. Abb. 1 (Holz); 3 (Glas); König, Fingerkunkel, Abb. 111g (Glas).

27 S. Jilek diskutiert für Beingriffe mit einem tetrastylen Mittelabschnitt eine 'Werkstatttradition' im kleinasiatischen Raum; S. Jilek in: Thür, Hanghaus B25. In diesen Rahmen kann man auch den Rocken KatNr. 2 stellen.

${ }_{28}$ KatNr. 10 ist oberhalb des Zapfens gebrochen, der Pinienzapfen sitzt deshalb nicht am äußersten Ende.

29 König, Fingerkunkel.

30 St. Karwiese, Das Beben unter Gallien und seine anhaltenden Folgen, in: Lebendige Altertumswissenschaften. Festschrift H. Vetters (1985) 126-131; Ladstätter, Chronologie 37.

31 Trinkl, Set; E. Trinkl, Vorbericht über die Versuchsgrabung im Abschnitt IV der Kathodos, in: D. Knibbe - G. Langmann (Hrsg.), Via Sacra Ephesiaca I, BerMatÖAI 3 (1993) 36-41. Zu den Funden aus dieser Grabung s. dies., Die Kleinfunde aus Sarkophag und Grabhaus an der Ostseite des Panayirdağ (Grabung 1991), in: D. Knibbe $-\mathrm{H}$. Thür (Hrsg.), Via Sacra Ephesiaca II, BerMatÖAI 5 (1995) 67-83.

32 Keiner unserer Spinnrocken - sofern er weit genug erhalten ist - zeigt jene späten Züge, die nach G. G. König und M. T. Bíró die Exemplare des 4. Jhs. kennzeichnen; König, Fingerkunkel; Bíró, Goddess.

33 Der aufwendige Arbeitsprozeß kann durch die Funde in den Hanghäusern aus verschiedenen Gründen nur punktuell beleuchtet werden. Ein Überblick zu Spinnen und Weben basierend auf den Funden des Hanghauses 1 bei Trinkl, Artefakte 313-327. - Zu einem Überblick über die in beiden Hanghäusern gefundenen Gegenstände für die Textilverarbeitung s. E. Trinkl, Artefacts Related to Preparation of Wool and Textile-processing Found inside the Terrace Houses of Ephesus, Turkey, in: C. Gillis - B. Nosch (Hrsg.), Ancient Textiles. Acta of the Conference 2003 (in Druck).

34 Bei sicherem Zugreifen sind die spitzen Blätter nicht nur hinderlich, sondern bei längerem Griff sogar schmerzhaft.

35 Auf Grund der wenigen bisher aus Ephesos bekannten Exemplare ist das Formenrepertoire der Verzierungen eingeschränkt. Anderenorts bekrönen Tiere - besonders häufig sind Vögel - oder Gefäße die Kunkeln. Insbesondere zur Diskussion der mit Vögeln bekrönten Kunkeln aus Bronze s. Gomolka-Fuchs, Spinngeräte; König, Fingerkun- 
Es ist daher notwendig, für diesen Typus der Spinnrocken, bei dem sowohl die Einzelelemente als auch der gesamte Aufbau des Geräts die Benutzung im Arbeitsprozeß des Spinnens ausschließen, eine andere Interpretation zu finden. Eine solche Erklärung muß allerdings zumindest mittelbar in einem Zusammenhang mit dem Produktionsprozeß des Spinnens stehen. Anderenfalls wäre die Entlehnung der Form von der Gestalt des Werkzeugs Spinnrocken unangebracht.

\section{Die Spinnrocken und das Hanghaus 2, dessen Räume und Bewohner}

Für jene Insula an der sog. Kuretenstraße, einer der Hauptverkehrsadern von Ephesos, die den modernen Namen Hanghaus 2 trägt, zeichnet sich stark komprimiert folgendes chronologisches Gerüst ab: Der auf dem Nordhang des Bülbüldağ, eines der beiden Stadtberge von Ephesos, liegende, für urbane Wohnzwecke genutzte Gebäudekomplex besteht aus ursprünglich mindestens sechs Wohneinheiten, die vom späten 1. Jahrhundert v. Chr. bis zu einer Erdbebenserie in den 60er Jahren des 3. Jahrhunderts $n$. Chr. mehrfach umgestaltet und restauriert wurden ${ }^{36}$. Die anschließende, bis in das 6. Jahrhundert erfolgte Weiternutzung des Areals steht mit dem ursprünglichen Bau und dessen Konzeption in nur losem Zusammenhang ${ }^{37}$, weswegen diese Zeit bei unseren Betrachtungen vernachlässigt werden kann.

Es werden im Folgenden lediglich jene Räume bzw. Raumgruppen kurz vorgestellt, die im Zusammenhang mit den besprochenen Spinnrocken von Bedeutung sind; immerhin stammen acht Exemplare gesichert von verschiedenen Stellen der Insula des Hanghauses 2. Teilweise wurden sie im unmittelbaren Zerstörungsschutt, teilweise im darüberliegenden Schuttmaterial gefunden. Ein Exemplar (KatNr. 3) stammt aus einem Kanal, so daß sein Fundort nicht ganz präzise zu bestimmen ist.

Keines der hier besprochenen Exemplare stammt aus der südöstlichsten Wohneinheit (WE 1) des Hanghauses 2. Vier Spinnrocken (KatNr. 1. 2. 3. 7) wurden hingegen in der auf derselben Terrasse westlich anschließenden Wohneinheit (WE 2) ${ }^{38}$ gefunden.

Die nördlich an die Wohneinheit 2 anschließenden, eine Terrasse tiefer liegenden Wohneinheiten 3 und $5^{39}$ sind - obgleich weitgehend ausgegraben - noch nicht detailliert untersucht, wodurch viele Fragen zu Baugeschichte, Chronologie und den Funden sowie zur Benennung der Räume noch offen sind. Ähnlich stellt sich auch die Situation in der Wohneinheit 7 dar $^{40}$. Dies ist in unserem Fall um so bedauerlicher, als aus dieser Wohneinheit zwei Spinnrocken (KatNr. 5. 6) stammen. Der Spinnrocken KatNr. 4, der aus dem Schutt im sog. Marmorsaal der Wohneinheit 6 stammt, dürfte jedoch ursprünglich, ebenso wie KatNr. 8, dem Bereich der Wohneinheit 4 zuzuordnen $\operatorname{sein}^{41}$. Für KatNr. 9 ist der Fundort nicht mehr zu eruieren, es scheint sich allerdings mit großer Wahrscheinlichkeit um eines der Hanghäuser zu handeln ${ }^{42}$.

kel; Gorny \& Mosch, Kunst der Antike 128. Auktion 13. Dez. 2003 (2003) 100 Nr. 450. Die Verfremdung der funktionalen Gestalt eines Rockens ist auch in anderen Materialien zu beobachten; W. Hagen, Kaiserzeitliche Gagatarbeiten aus dem rheinischen Germanien, BJb 142, 1937, bes. 135 f. Taf. 34; R. Pirling, Klothos Kunkel, in: Th. E. Haevernick - A. v. Saldern (Hrsg.), Festschrift W. Haberey (1976) 101-109; Trinkl, Set.

36 Der Bautypus, der den einzelnen Wohneinheiten zugrundeliegt, auch wenn die Strukturen durch Ein- und Umbauten teilweise verunklärt sind, ist der Typus des Peristylhauses. Zur Datierung s. Ladstätter, Chronologie 33 ff.

37 G. Wiplinger, Die Bauphasen der Wohneinheiten 1 und 2, in: Krinzinger, Baugeschichte $84 \mathrm{ff}$.

$38 \mathrm{Zu}$ den Bauphasen der Wohneinheit 1 und 2 s. E. Rathmayr in: Krinzinger, Wohneinheiten; S. Ladstätter in: Krinzinger, Wohneinheiten; vgl. o. Anm. 8.

39 Die benachbarten Wohneinheiten 3 und 5 wurden in den Jahren 1972-1975 freigelegt; H. Vetters, AnzWien 110, 1973, 186 ff.; ders., AnzWien 111, 1974, 219; ders., AnzWien 113, 1976, 496 Abb. 4. Zu den Nachuntersuchungen s. S. Ladstätter, ÖJh 70, 2001, 255 f.; Ladstätter, Chronologie 33 ff.

40 Ladstätter, Chronologie 38.

${ }^{41}$ H. Thür in: Thür, Hanghaus $101 \mathrm{f}$.

${ }^{42}$ Im Hanghaus 1 kam kein vergleichbares Exemplar zutage; das dürfte mit der über das 3. Jh. hinausreichenden Benutzungszeit der Wohnhäuser zusammenhängen; Trinkl, Artefakte. 
Betrachtet man die Fundorte der einzelnen Spinnrocken genauer, so ergeben sich manche Konzentrationen in bestimmten Bereichen der Wohneinheiten 2, 4 und 7. Obgleich die Gesamtzahl von acht zu lokalisierenden Exemplaren sehr klein ist, scheint mir diese Verteilung kaum zufällig zu sein.

Die Venuskunkel KatNr. 1 kann der unmittelbaren Zerstörungsschicht in SR 27 der Wohneinheit 2 zugerechnet werden ${ }^{43}$. SR 27 ist der Hofbereich, über den man das Peristyl und die anschließenden repräsentativen Räume erreichte. Es handelt sich einerseits um einen Durchgangsbereich, andererseits um einen Raum, dem unterschiedliche Formen der Nutzung zugeschrieben werden können; der öffentliche Aspekt spielt aber zweifelsohne in Anbetracht der Lage innerhalb des Raumgefüges eine große Rolle. Die Fundumstände der Venuskunkel KatNr. 1, »in Bodennähe « ${ }^{44}$, lassen auf eine ursprüngliche Aufbewahrung in unmittelbarer Nähe des Hofes bzw. in SR 27 selbst schließen. Etwas weniger präzise ist der Fundort des Rockens KatNr. 2 zu benennen: im Schutt von SR 27a, der nachträglich an der Südostecke des Hofes SR 27 eingebauten Küche der Wohneinheit 2. Aus der Grabungsgeschichte des Hanghauses 2 und aus vergleichbaren Fällen in derselben Insula läßt sich schließen, daß die Zuordnung zu SR 27a nicht genau erfolgen kann ${ }^{45}$. Es könnte sich auch um Sturzmaterial aus einem Obergeschoß im Bereich des Hofes SR 27 handeln. Und noch weniger genau kann der ursprüngliche Aufbewahrungsort von Rocken KatNr. 3 eruiert werden. Für dieses Rockenfragment ist der Kanal in SR 27c, an der Nordwestecke des Hofes SR 27, als Fundort verzeichnet. Dieser führt von Süden durch SR 27c und entwässert in die Stiegengasse nach Westen. Der Rocken KatNr. 3 könnte natürlich im Kanal mitgeschwemmt worden sein ${ }^{46}$. Es liegt hier allerdings eine unerwartete Übereinstimmung mit den Fundorten der Rocken KatNr. 1 und KatNr. 2 vor, so daß wohl auch in diesem Fall der ursprüngliche Platz im Einzugsgebiet von SR 27 bzw. dem dazugehörigen Oberstock liegt. Die Fundangaben für den Rocken KatNr. 7 sind leider widersprüchlich, gesichert ist lediglich die Wohneinheit $2^{47}$.

Zwei Exemplare aus der Wohneinheit 7 stammen wiederum aus dem unmittelbaren Einzugsgebiet des Peristylhofes, allerdings von nahezu gegenüberliegenden Seiten: KatNr. 5 wurde in R 38e gefunden, der nördlich an den nördlichen Peristylumgang anschließt $\mathrm{t}^{48}$; KatNr. 6 stammt aus einem Schuttstratum ${ }^{49}$ in einem an der Südostecke des Peristyls der Wohneinheit 7 liegenden Raum (R 38a).

Ähnlich schwierig stellt sich auch die Situation für die beiden der Wohneinheit 4 zugeordneten Spinnrocken dar: Das heute leider verschollene Rockenfragment KatNr. 8, das gemeinsam mit Skulpturbruchstücken und Fragmenten von Ausstattungsgegenständen gefunden wurde ${ }^{50}$, stammt aus dem Schutt der Wohneinheit 4 und kann keiner Raumeinheit zugeordnet werden ${ }^{51}$. Für die gemeinsam mit dem abgestürzten Material aus der Wohneinheit 4 im sog. Marmorsaal

43 Summarisch zur Baugeschichte: G. Wiplinger, Die Bauphasen der Wohneinheiten 1 und 2, in: Krinzinger, Baugeschichte 65. 74. Die Zerstörung des Hofes gibt mit etwa $270 \mathrm{n}$. Chr. einen terminus ante quem für die Entstehung der Fingerkunkel; E. Rathmayr in: Thür, Hanghaus.

$44 \mathrm{Zu}$ den unterschiedlichen Mitfunden s. Katalog.

45 Ladstätter, Chronologie 12 ff.; dies. in: Krinzinger, Wohneinheiten.

46 Als Beifunde sind fünf Beinnadeln und eine Nähnadel verzeichnet.

47 Als Fundort kommen entweder SR 21, Oberstock südlich von SR 25 bzw. dessen Oberstock (H. Vetters, AnzWien 107, 1970, 111 Abb. 3 ff.) oder SR 27 in Frage. In Anbetracht der Fundorte der Kunkeln KatNr. 1-3 scheint eine Zuordnung zum Schutt in SR 27 etwas plausibler.

48 H. Vetters, AnzWien 119, 1982, 74.

49 Die Verschüttung von R 38a dürfte allerdings der Zerstörung der Wohneinheit 7 vorausgehen. R 38a war ebenso wie der angrenzende Raum R 38c bei der endgültigen Zerstörung aufgeschüttet; H. Vetters, AnzWien 118, 1981, 148; ders. (Anm. 48) 74. Da es sich in diesem Fall wohl um eine intentionelle Einfüllung handelt, ist die Anwesenheit von KatNr. 6 insofern problematisch, als die Einfüllung von Wert- bzw. Prestigeobjekten in diese Aufschüttung als ungewöhnlich einzustufen ist.

50 s. Anm. 21.

${ }^{51}$ Gestürztes Material aus einem der drei Obergeschosse wäre ebenso denkbar; H. Thür in: Thür, Hanghaus 101. 
der Wohneinheit 6 gefundene Fingerkunkel KatNr. 4 kann an eine Zuordnung in den nördlichen Bereich der Wohneinheit 4 gedacht werden, am ehesten an eines der Obergeschosse. Dieser Bereich zählte in der letzten Bauphase des Hanghauses $2 \mathrm{zu}$ den repräsentativen Wohnräumen der Wohneinheit $4^{52}$.

Obwohl die Fundumstände zugegebenermaßen nicht gesichert sind, ist die Frage zu stellen, $\mathrm{ob}$ in der Verteilung der Spinnrocken eine gemeinsame Linie zu erkennen ist. Es fällt auf, daß es - obgleich es sich typologisch um ein Werkzeug handelt - keine charakteristische Vergesellschaftungen mit anderen Werkzeugen gibt, weder mit anderen Werkzeugen für die Textilverarbeitung (naturgemäß müßten Spindel, Spinnwirtel und Rocken eigentlich ein funktionales Set darstelle ${ }^{53}$ ) noch mit Werkzeugen für andere Funktionen. Die mögliche Erklärung dafür könnte in den beschriebenen Mißproportionen liegen, die eine Verwendung dieser Spinnrocken im Arbeitsprozeß ausschließen. Sie werden dadurch zu funktionslosen Gegenständen, deren tieferer Sinn erst aufzuspüren ist.

Auch die Bereiche der einzelnen Wohneinheiten, welchen oben - so gut es die Umstände erlauben - die Spinnrocken zugeordnet wurden, sind nicht jene Räumlichkeiten, in denen man sich traditionellerweise verstaute Gerätschaften oder deponierte Werkzeuge vorstellt ${ }^{54}$ : der Hof der Wohneinheit 2 (SR 27), die Räume in der Nähe des Peristyls der Wohneinheit 7 (R 38) und wahrscheinlich repräsentative Räume im Oberstock der Wohneinheit $4^{55}$. Um so mehr drängt sich die Frage nach dem Sinngehalt der Spinnrocken auf.

An die aus dem Hanghaus 2 stammenden Spinnrocken werden hier fünf weitere fragmentarisch erhaltene Exemplare desselben Typus angeschlossen, die von anderen Fundplätzen in Ephesos stammen, sowie ein gut erhaltenes Exemplar, das vom Efes Müzesi in Selçuk angekauft wurde. KatNr. 10 wurde zusammen mit Spindel und Spinnwirtel in einem Sarkophag des zweiten Viertels des 3. Jahrhunderts an der Damianosstoa gefunden ${ }^{56}$; bei dieser Grablegung handelt es sich um die Bestattung einer schwangeren Frau und eines Kleinkindes ${ }^{57}$. Auch die übrigen Grabbeigaben (Schmuck, Spiegel, Bronzebüchse, Glasgefäße, Beinnadeln) fügen sich in das Bild vergleichbarer Grablegungen ein. Sie lassen ebenso wie der steinerne Sarkophag und die prominente Lage der Bestattung in der zum Zeitpunkt der Bestattung neu errichteten Prozessionshalle auf eine keineswegs arme Familie schließen ${ }^{58}$. Welche Rolle der maximal 35jährigen Verstorbenen in dieser Familie jedoch zukam, muß derzeit offenbleiben.

Der Rocken KatNr. 14 wurde in einem Kanal östlich des Südturmes des Magnesischen Tores gefunden, in dem er mit einer Münze des Constantius II. vergesellschaftet war ${ }^{59}$. Bereits in den

52 Ebenda.

53 J. P. Wild, Textiles in Archaeology (1988) 25 ff.; Trinkl, Set.

${ }^{54}$ Zur Lagerung von Geräten und Werkzeugen im Hanghaus 1 s. S. Jilek, Die Metall- und Beinfunde, in: LangAuinger, Hanghaus 254 ff.; dies., Mobiliar aus den Hanghäusern in Ephesos, in: B. Asamer - W. Wohlmayr (Hrsg.), Akten des 9. Österreichischen Archäologentages (2003) 87-91. - Im Hanghaus 1 wurde ein deponiertes Set aus Webgewichten im Raum E2 gefunden; Trinkl, Artefakte, bes. 320 f.

${ }_{55} \mathrm{Zu}$ den Obergeschossen über dem sog. Sokrateszimmer s. H. Thür in: Thür, Hanghaus 101; N. Zimmermann in: Thür, Hanghaus.

56 D. Knibbe, Der Verlauf der Via Sacra und ihre Überdachung durch T. Flavius Damianus, in: Knibbe - Langmann (Anm. 31) 16-27; Trinkl (Anm. 31:1993) 36-41; Trinkl, Set; D. Knibbe, Topographica Ephesiaca. Damianosstoa - Androklosgrab - Olympieion und Koressos, ÖJh 71, 2002, 207-219. - Zu jüngsten Untersuchungen weiterer Grablegungen unweit davon s. M. Steskal u. a., Die Damianosstoa in Ephesos. Bericht über die Ausgrabung 2002 im Abschnitt Kathodos III, ÖJh 72, 2003, 241 ff.

57 E. Reuer - S. Fabrizii-Reuer, Die Ergebnisse der anthropologischen Untersuchung der aus Sarkophag und Grabhaus geborgenen menschlichen Skelette, in: Knibbe - Langmann (Anm. 31) 43 f.

58 Der Sarkophag trug weder Dekor noch Inschrift; die auf der Frontseite nicht mittig angebrachte tabula ansata trug ursprünglich wohl ein Dipinto, das den Namen der Grabinhaberin wiedergab. Der ohnehin große Sarkophag sollte den in der Halle stehenden Betrachtern durch den aus der Mitte verschobenen First des Deckels optisch noch größer erscheinen; D. Knibbe, Der Verlauf der Via Sacra und ihre Überdachung durch T. Flavius Damianus, in: Knibbe - Langmann (Anm. 31) $22 \mathrm{f}$.

59 G. Seiterle danke ich für die präzisen Angaben zur Auffindung des Rockenfragmentes. 
60er Jahren wurden bei der Untersuchung der Basilika auf dem Staatsmarkt die drei teilweise relativ kleinen Bruchstücke KatNr. 11-13 gefunden; präzise Fundumstände sind heute jedoch schwierig zu eruieren ${ }^{60}$. Über Beifunde und Fundumstände sind wir auch bei dem angekauften Stück KatNr. 15 nicht unterrichtet; der gute Erhaltungszustand würde aber auch in diesem Fall, ebenso wie bei den Spinnrocken KatNr. 10 und 14, an eine Herkunft aus dem sepulkralen Bereich denken lassen.

\section{Pflichten und Freiheiten römischer Frauen}

Das römische Gesellschaftssystem, auch jenes in Kleinasien im 2. und 3. Jahrhundert n. Chr., basiert auf der Klientelwirtschaft: der patronus/dominus sorgt sich um seine clientes, die im Gegenzug ihm und seiner Familie standesgemäßes Leben ermöglichen. Dieses Verhältnis kommt in der allmorgendlichen Vorsprache, salutatio, der Klienten zum Ausdruck und äußert sich im Empfang des patronus bei dieser und bei anderen Gelegenheiten. Beim Empfang und bei der Organisation seiner Klientel wird der Hausherr von einem nomenclator unterstützt; im allgemeinen werden Höfe, Atria und Peristyle als Räumlichkeiten für die wartende Klientel angesprochen. Die Audienz erfolgt in einem dem Warteraum angeschlossenen Zimmer ${ }^{61}$. Am abendlichen Gastmahl, cenatio, in einem der Repräsentationsräume der domus, nimmt auch die domina teil ${ }^{62}$.

Die Grundrisse der Wohnhäuser im gesamten Römischen Reich spiegeln diese Gesellschaftsordnung wider: in ihnen ist ein entsprechend großer Anteil der Grundfläche für die Nutzung als öffentlicher Raum konzipiert, der von J. A. Dickmann u. a. als Anlage für die repräsentative Nutzung beschrieben wird $^{63}$. M. Trümper bewertet die repräsentativen Räumlichkeiten der Häuser auf Delos hierarchisch ${ }^{64}$.

Die Differenzierung einzelner Räume kann auch in den Hanghäusern in Ephesos im Grundriß nachvollzogen werden und spiegelt sich in der baulichen Ausstattung (Malerei, Marmorwandverkleidung, gemalte Marmorwandverkleidung etc.) und wahrscheinlich auch in der beweglichen Ausstattung wider ${ }^{65}$. Zu den mit Marmorwandverkleidung, Marmorinkrustation und mit reicher Malerei ausgestatteten Räumen ist im Hanghaus 2 in Ephesos noch der Typus des seitlich an den Hof bzw. an das Peristyl angebauten Musenzimmers zu den repräsentativen Räumen zu zählen ${ }^{66}$.

${ }^{60}$ Das Vergleichsmaterial zu KatNr. 11 läßt vermuten, daß die Form der Rocken ohne Verzierungen am Übergang von Schaft und Ringöse älter als die reich dekorierten Exemplare aus dem Hanghaus 2 ist. Zur Form einer für das 1. Jh. n. Chr. charakteristischen Spindel s. S. Martin-Kilcher, Geräte und Geräteteile aus Knochen und Hirschhorn aus dem Vicus Vitudurum-Oberwinterthur, Vitudurum 5 (1991) bes. 64.

${ }^{61}$ Zur Verbindung von salutatio und den Räumen, in denen diese stattfand, vgl. Dickmann, Domus 51 f. - Zur Veränderung des Grades der 'öffentlichen Privatsphäre' in bezug auf das Gastmahl s. S. P. Ellis, Late-antique dining: architecture, furnishings and behaviour, 22. Suppl. JRA (1997) 41-51.

62 Dickmann, Domus 313 ff.; Ch. Rohweder, Eine römische Dichterin. Sulpicia, in: Späth - Wagner-Hasel, Frauenwelten 147-161; K. Bradley, The Roman Family at Dinner, in: I. Nielsen - H. S. Nielsen (Hrsg.), Meals in a Social Context (1998) 36-55.

63 J. A. Dickmann erkennt in Pompeji eine ab dem späten 2. Stil einsetzende Entwicklung, bei der die im Rahmen von Neu- oder Umbauten bzw. nach dem Ankauf eines Nachbargrundstückes errichteten Cenatio-Cubiculum-Gruppen die zuvor weitverbreiteten Triclinum-Cubiculum-Gruppen ablösen. Dabei eifern die Hausherren in den Vesuvstädten den kaiserlichen cenae rectae nach; Dickmann, Domus 313 ff.; die entsprechenden literarischen Quellen zusammengestellt ebenda 321 Anm. 129. - Auf Grund der unterschiedlichen Sozialstruktur ist das griechische Haus anders als das römische aufgebaut; K. Reber, Aedificia Graecorum. Zu Vitruvs Beschreibug des griechischen Hauses, AA 1988, 653-666.

64 Raumgruppen werden von M. Trümper in Gruppen zu oecus maior und oecus minor zusammenfaßt. Auch wenn diese strikte Trennung nicht bis ins letzte Detail bei jedem Haus schlüssig nachvollziehbar ist, wird die unterschiedliche Wertigkeit der Repräsentativräume deutlich; M. Trümper, Wohnen in Delos. Eine baugeschichtliche Untersuchung zum Wandel der Wohnkultur in hellenistischer Zeit, Internationale Archäologie 46 (1998) 15 ff.

${ }_{65}$ Krinzinger, Baugeschichte; Lang-Auinger, Hanghaus; Thür, Hanghaus.

66 N. Zimmermann, Ausstattung von Haupt- und Nebenräumen. Zur Datierung der Wandmalereien des Hanghauses 2 in Ephesos, in: Krinzinger, Baugeschichte 107 ff.; D. Parrish, House (or Wohneinheit) 2 in Hanghaus 2 at Ephesos: 
Aber wodurch wird die domus charakterisiert? Sie bedeutet sicherlich mehr als den Aufenthaltsort der Familie, der auch in einer Villa am Stadtrand oder auf dem Land gelegen sein kann. In der juristischen Fachliteratur stellt sich die domus als rechtlich separierte Zone dar, aus deren Bereich man weder durch eine gerichtliche Vorladung noch durch Gewalt entfernt werden konnte; dieses Gesetz war allerdings nicht an den persönlichen Besitz gebunden ${ }^{67}$. Wollte man einer Person Schaden zufügen, veranlaßte man einen physischen oder auch nur imaginären, beispielsweise durch Lärm oder Angriff auf die Haustür (flagitatio); der Besitzer hatte aber seinerseits das Recht, sein Haus auch mit handfesten Mitteln zu verteidigen ${ }^{68}$.

Die Türschwelle und das Eingangstor markieren in jedem Fall eine deutliche Trennung zwischen öffentlichem und privatem Besitz, zwischen einem im Besitz der Allgemeinheit stehenden Areal und einem Bereich, für den eine entsprechende Ein- oder auch Vorladung notwendig ist. Das gesamte Haus ist ein Bereich limitierten Zugangs, abgesondert von der Welt auf der Straße und gilt in der römischen Vorstellungswelt als sanctus ${ }^{69}$.

Diesem Hausbereich, domus, werden rechtlich alle sich darin befindenden beweglichen und unbeweglichen Güter zugeordnet sowie jene Personen, unabhängig von ihrem Stand, die das Haus bewohnen. Die Fürsorge für das Haus, abgesehen vom juridischen Schutz, oblag der Hausfrau, der domina ${ }^{70}$ : bei der Hochzeit wird die Ehefrau, matrona, zur Wächterin des Hauses ${ }^{71}$.

Das römische Eherecht sieht strenge Güterteilung vor, so daß eine verheiratete Frau, sofern sie durch die Familie oder durch selbständigen Erwerb Besitz hatte, diesen durch eine Eheschließung nicht einbüßte; sie konnte auch weiterhin darüber verfügen, wie es indirekt durch die dokumentierten Streitfälle nach Schenkungen durch Frauen belegt ist ${ }^{72}$. Im Fall des Todes eines Ehepartners ging - von testamentarischer Ordnung abgesehen - der Besitz des Verstorbenen nicht automatisch in das Eigentum des überlebenden Ehepartners über, sondern blieb im Besitz der Familie ${ }^{73}$. Sogar

A Few Issues of Interpretation, in: H. Friesinger - F. Krinzinger (Hrsg.), 100 Jahre Österreichische Forschungen in Ephesos. Akten des Symposions Wien 1995, AForsch 1, DenkschrWien 260 (1999) 507-513.

67 Treggiari, History $83 \mathrm{ff}$.

68 Treggiari, History 95 ff.

69 Treggiari, History $74 \mathrm{ff}$.

$70 »$ Römerinnen haben eine traditionelle und ganz selbstverständliche Präsenz in der domus und verfügen in diesem gesellschaftlichen Raum über weitreichende Kompetenzen. [...] Die domus als Handlungsraum von Frauen - als Gattinnen, Töchter oder Mütter - umfaßt die Verwandtschaft im weitesten Sinn genauso wie die sozialen Netze von Freundschaft und Patronage.« Th. Späth, Skrupellose Herrscherinnen? Das Bild der Agrippina minor bei Tacitus, in: Späth - Wagner-Hasel, Frauenwelten, bes. 272. 274.

71 Das Symbol dafür ist oft der Schlüssel oder Schlüsselbund; Cic. Phil. 2, 69. T. E. V. Pearce, The Role of the Wife as CUSTOS in Ancient Rome, Eranos 72, 1974, 16-33; S. Treggiari, Roman Marriage. Iusti coniuges from the time of Cicero to the time of Ulpian (1991). - Durch ihre Hochzeit wird eine Frau zur Herrin des Hauses, domina, und übernimmt dessen Betreuung. Ihre zweite Hauptaufgabe sind Geburt und Aufzucht der Kinder, matrona. Deshalb erbaten die Frauen Roms alljährlich am 1. April im Tal des Circus Maximus mit Gebeten an Venus Verticordia und Fortuna Virilis die erfolgreiche Erfüllung des ehelichen Sexuallebens; zusammenfassend bei J. Scheid, Die Rolle der Frauen in der römischen Religion, in: P. Schmitt Pantel (Hrsg.), Geschichte der Frauen I. Antike (1993) bes. $427 \mathrm{ff}$. In der Beschreibung der Frauen in Tacitus' Annalen sind pudicitia und fertilitas die zwei wichtigsten Pflichten einer Ehefrau; P. Setälä, Female Property and Power in Imperial Rome, in: Larrson Lovén - Strömberg, Women, bes. 97. - Besonders die Sorge der Frauen um den Nachwuchs sieht M. T. Bíró in den Venuskunkeln repräsentiert; die Venusstatuetten der Prunkrocken sind nach Bíró primär Darstellungen der Aspekte von Muttergottheiten; Bíró, Goddess, passim. Ähnlich auch im Zusammenhang mit Isis lactans bei Gomolka-Fuchs, Spinngeräte. - In der militärischen Umgebung hatten naturgemäß ausschließlich Männer die Schlüsselgewalt, wie H. Ubl anhand eines mit Besitzerinschrift versehenen Schlüssels im Museum Lauriacum jüngst wieder kurz diskutierte; H. Ubl, Ein Schlüssel, ein obszöner Begriff und ein Centurio, MMVLaur 37, 1999, 5-10.

72 J. F. Gardner, Family and Familia in Roman Law and Life (1998) 220 ff. 233 ff.

73 J. U. Krause, Wirtschaftliche und gesellschaftliche Stellung von Witwen (1994) 174 ff. In Ägypten ist ab dem 2. Jh. die frontistria belegt; bei ihr handelt es sich um eine Frau, die das Sorgerecht für ihre Kinder übernommen hatte; V. Vuolanta, Women and Property of Fatherless Children in the Roman Empire, ActaInstRomFin 25 (2002) 203-243 bes. 218 ff. - Es muß allerdings festgehalten werden, daß nachgewiesenerweise Töchter in der Erbfolge oft benachteiligt wurden; J. Pölönen, The Division of Wealth between Men and Women in Roman Succession, ActaInstRomFin 25 (2002) 147-179. 
M. R. Lefkowitz muß eingestehen, daß die römischen Frauen mehr Rechte vor dem Gesetz hatten, als die meisten tatsächlich ausnutzten ${ }^{74}$.

Wir kennen aus dem gesamten Römischen Reich Grundeigentümerinnen und Besitzerinnen von Firmen sowie Pächterinnen entsprechender Betriebe; in der Kaiserzeit sind sie sogar häufiger als davor und danach belegt ${ }^{75}$. Besonders auffallend ist die große Zahl weiblicher Gutsbesitzerinnen aus senatorischen Familien im 2. Jahrhundert n. Chr., viele sind uns durch ihre Namen auf Ziegelstempeln überliefert; diese Frauen verfügten auch über entsprechende Fabriken, Angestellte und Sklaven. Sie engagierten aber üblicherweise einen Manager, officiniator, für die Betreuung dieser Güter, wobei diese Funktion allerdings wiederum von einer Frau, officinatrix, ausgeübt werden konnte ${ }^{76}$. Frauen sind uns darüber hinaus sowohl als Darlehensnehmerinnen als auch als Gläubigerinnen überliefert; J. U. Krause vermutet sogar, daß reiche Römerinnen diese Art des Gelderwerbs Grundgeschäften oder dem produzierenden Gewerbe vorzogen, um ihre Geschäfte nicht allzu öffentlich werden zu lassen ${ }^{77}$.

Die Aufteilung der römischen Gesellschaft in einen privaten Bereich der Frauen im Haus und einen öffentlichen Bereich außer Haus für die Männer kann heute als überholt angesehen werden; T. Scheer ist überzeugt: »Inzwischen muß man die Geschichtsfähigkeit der antiken Frauen nicht mehr beweisen. ... (es) muß die Betrachtung des historischen und sozialen Ganzen, von zwei Geschlechtern gebildet, im Mittelpunkt stehen. « $^{78}$

Wenn nun die domina/matrona der gehobenen Gesellschaft ökonomische und gesellschaftliche, vielleicht sogar politische Verpflichtungen hatte, ähnlich wie der Hausherr, so drängt sich die Frage nach den entsprechenden Räumlichkeiten, die ihr dafür zur Verfügung standen, auf.

Zumindest für Livia war ihre große Klientel sprichwörtlich und vor allem nach dem Tod des Augustus unverzichtbar ${ }^{79}$. Auch wenn manche Kaiserinnen besonderen Wert auf das traditionelle Frauenbild legten, wie z. B. die Gattin Traians, Plotina, war der Einfluß auf ihren Mann/Sohn und Regenten meist sehr groß. Er nimmt im Lauf der Kaiserzeit noch stetig zu: die Ehefrau

74 M. R. Lefkowitz, Wives and Husbands, in: I. McAuslan - P. Walcot (Hrsg.), Women in Antiquity (1996) 67-82 bes. 79 .

75 Krause (Anm. 73$) 198$ ff.; R. MacMullen, Women's Power in the Principate, Klio 68, 1986, 434-443. - Aurelia Charite, Grundbesitzerin im Ägypten des 4. Jhs., bezeichnet sich selbst in einer Pachturkunde als »schreibkundig« und »ohne Rechtsvormund auftretend«. Dies trifft auch auf andere, aber nicht alle Frauen zu, die uns aus den Papyri bekannt sind; H. Harrauer, Großgrundbesitzerinnen nach den Papyrusquellen, in: E. Specht (Hrsg.), Frauenreichtum. Die Frau als Wirtschaftsfaktor im Altertum (1994) 181-197.

76 Setälä (Anm. 71) bes. 101 ff. Setälä ist überzeugt: »The female dominae, and also the female officinatrices, demonstrate female leadership and entrepreneurship.«; dies., Women and Brick Production - some new Aspects, ActaInstRomFin 25 (2002) 181-201. Entsprechende Bildung darf und muß bei ihnen vorausgesetzt werden. - Um Bildung und Intelligenz einer Frau ranken sich Anekdoten, diese Eigenschaften finden auch in Grabinschriften Erwähnung; s. u. Anm. 81 (Burckhardt). Neben den 'alten' weiblichen Qualitäten müssen Ehefrauen unbedingt auch Geometrie und Philosophie beherrschen, um ihren Geist höheren Dingen zuzuwenden; Plut. mor. 145c-d.

77 Krause (Anm. 73) 190 ff. In unserem Zusammenhang illustrativ ist auch die Sonderstellung jener mit lanifica bezeichneten Frauen unter den Arbeiterinnen mit Wolle, wie sie die epigraphischen Quellen belegen; M. Eichenauer, Untersuchungen zur Arbeitswelt der Frau in der römischen Antike (1988) 90. Es werden offensichtlich nur matronae mit diesem Begriff benannt.

78 Die entsprechende Literatur zusammenfassend bei T. Scheer, Forschungen über die Frau in der Antike. Ziele, Methoden, Perspektiven, Gymnasium 107, 2000, 143-172 bes. 166 ff. - Schwierig zu lokalisieren sind auch Räumlichkeiten für Dienerschaft und Sklaven, deren Existenz auf Grund der Gesellschaftsordnung außer Frage steht; M. George, Servus and domus: the Slave in the Roman House, 22. Suppl. JRA (1997) 15-24: Etliche literarische Quellen belegen jedoch, daß die Sklaven am Ort ihrer Dienstausübung schlafen, so daß Quartiere für Sklaven überhaupt nicht zu erwarten sind; George a. O. bes. 22 Anm. 41.

79 Livia, die Gattin und Witwe des Augustus, führt einen großen Haushalt mit entsprechenden öffentlichen und privaten Verpflichtungen, jedoch ohne daß er - wie für die spätere Zeit charakteristisch - als übersteigert empfunden werden kann. Über das in ihrem Haus arbeitende Personal sind wir teilweise sehr gut durch das Monumentum Liviae, das große Columbarium an der Via Appia, unterrichtet. Die Grabinschriften der z. T. an diesem Ort bestatteten Dienerschaft geben einen Einblick in die differenzierte Struktur des Haushalts der Livia. S. Treggiari, Jobs in the Household of Livia, BSR 43, 1975, 48-77. 
sowie die gesamte Familie begleiten den Kaiser auch auf Reisen in die Provinzen, wodurch sie gleichzeitig als Unterstützung und als Demonstration des Herrschafts- und Nachfolgeanspruchs fungieren ${ }^{80}$.

Bereits das Verhalten von Ciceros Frau Terentia, die ihr beträchtliches Vermögen auch nach der Eheschließung selbst verwaltete, macht deutlich, daß entsprechende Räumlichkeiten für die administrative Tätigkeiten der matrona im Bauplan des Hauses vorgesehen gewesen sein mußten. Vergleichbares Handeln wird auch von den Ehefrauen der begüterten Farmer erwartet ${ }^{81}$. Das Zusammentreffen mit einer kleineren oder größeren Anzahl von Personen in ihrem Haus ist dafür unerläßlich ${ }^{82}$. Diese in aller Kürze angesprochenen Beispiele belegen, daß Römerinnen aktiv im Berufs- und Gesellschaftsleben standen.

\section{Die Frauen in der Oberschicht Kleinasiens}

Wie sehr die römischen Städte, darunter auch Ephesos, auf die Mitgestaltung des städtischen Erscheinungsbildes und die (monetäre) Präsenz, auch von Frauen, angewiesen waren, zeigen nicht nur die von ihnen errichteten Bauten, sondern ebenso die lange Liste von Frauen im Amt von Gymnasiarchen, als Kaiserpriesterinnen und als Trägerinnen von Leiturgien. F. Quaß kann sich sogar eine auf dem entsprechenden Vermögen basierende, verpflichtende Leiturgie (auch außerhalb der religiösen Sphäre) vorstellen ${ }^{83}$. Die Übernahme derselben Aufgaben auch durch Kinder reduziert jedoch die Bedeutung der Eigenverantwortlichkeit der Frauen in diesen Ämtern $^{84}$. Auch wenn es das private bzw. das Familienvermögen ist, das diese Frauen für ihre 'Karriere' qualifizierte ${ }^{85}$, so finden diese Leistungen doch in der Öffentlichkeit statt und die verpflichtete(?) Wohltäterin tritt an die Stelle eines Mannes oder zumindest mit gewisser Eigenverantwortung an die Seite ihres Mannes oder eines anderen nahen Verwandten.

Besonders aus dem südlichen Kleinasien ${ }^{86}$ kennen wir einige prominente Frauen, die in den genannten Funktionen und Ämtern der Pflicht ihrer Familie nachkommen, auch wenn J. Nollé

${ }^{80}$ H. Halfmann, Itinera principium, Heidelberger althistorische Beiträge und epigraphische Studien 2 (1986) 90 ff.; M. Th. Raepsaet-Charlier, Epouses et familles de magistrats dans les provinces romaines aux deux premiers siècles de l'Empire, Historia 31, 1982, 56-69; M. Keltanen, The Public Image of the Four Empresses - Ideal Wives, Mothers and Regents?, ActaInstRomFin 25 (2002) 105-146. E. R. Varner, Portraits, plots, and politics. Damnatio memoriae and the images of imperial women, MemAmAc 46, 2001, 41-93.

${ }_{81}$ Treggiari (Anm. 79) bes. 63 Anm. 152: Col. 12 pr. 1 ff.; Plut. Cato maj. 20, 3. - Die hohe Intelligenz hebt Cicero auch bei seiner Tochter Tullia hervor, jedoch nicht ohne sie mit seiner eigenen zu vergleichen; Treggiari, History 58. - Entsprechende Bildung der Mütter fordert Quintilian (Quint. inst. 1, 1,6) für eine gelungene Erziehung der Kinder; als Beispiel dient ihm Cornelia, die Mutter der Gracchen. Zum politischen Einfluß der Cornelia vgl. L. Burckhardt - J. von Ungern-Sternberg, Cornelia, die Mutter der Gracchen, in: Dettenhofer, Männersache 97-132.

82 A. Wallace-Hadrill, Engendering the Roman House, in: D. E. E. Kleiner - S. B. Matheson (Hrsg.), I Clavdia. Women in Ancient Rome (1996) 104-115.

${ }^{83}$ F. Quaß, Die Honoratiorenschicht in den Städten des griechischen Ostens (1993) 321. 338. Zu Frauen als Bauherren in Ephesos s. u. - Seit der Kaiserzeit wird den Frauen, nicht zuletzt auf Grund der unter Augustus erlassenen leges Iulia et Papia, zunehmend mehr Eigenständigkeit zugestanden, so daß sich sowohl rechtliche als auch geübte Lebensnormen von den griechischen deutlich unterscheiden; Gai. inst. 1, 145. 190.

${ }^{84}$ Quaß (Anm. 83). In diesem Zusammenhang muß auch die Stiftung der Eumachia in Pompeii, wahrscheinlich zugunsten der Gilde der Wollhändler erwähnt werden; W. O. Mueller, The Wool Trade of Ancient Pompeii (1976) $57 \mathrm{ff}$.

85 R. van Bremen, Women and Wealth, in: A. Cameron - A. Kuhrt (Hrsg.), Images of Women in Antiquity (1983) 223-242; Scheer (Anm. 78) bes. $158 \mathrm{f}$.

86 Die Frauen mancher Regionen Kleinasiens zeigen bereits in hellenistischer Zeit größere Eigenständigkeit als ihre Geschlechtsgenossinnen in anderen Gebieten; J. Fabricius, Gelagerte Frauen. Überlegungen zu einer ikonographischen Besonderheit rhodischer Totenmahlreliefs und zur sozialen Stellung der Frau im hellenistischen Rhodos, in: POAOE. 2400 XPONIA (1999) 207-214; R. Merkelbach - J. Stauber (Hrsg.), Steinepigramme aus dem griechischen Osten I (1998) 451. Überblick zu den weiblichen Gymnasiarchen in Kleinasien bei L. Cassarico, Donne gimnasiarco, ZPE 48, 1982, 117-123. Die Ehrenstatuen für Frauen Kleinasiens hellenistischer Zeit tragen jedoch 
darin in Ermangelung eines männlichen Nachkommens oder auf Grund der Abwesenheit des Mannes nur eine Art 'Verlegenheitslösung' sehen möchte ${ }^{87}$ : Plancia Magna in Perge, Motoxaris aus Selge, Menodora in Sillyon. Sie bekleiden sogar die eponymen Ämter in ihren jeweiligen Heimatstädten.

In einer Ehreninschrift aus der Mitte des 1. Jahrhunderts n. Chr. lobt die Stadt Patara ihre Bürgerin Iunia Theodora, die Land besaß und die in politischer und wirtschaftlicher Mission selbst nach Korinth reiste. Besonders wird ihre Großzügigkeit zugunsten der Stadt und deren Bürger herausgestrichen ${ }^{88}$. In unserem Zusammenhang ist gerade die Erwähnung, auf welche Weise Iunia Theodora ihren öffentlichen Umgang pflegte, von Bedeutung: sie empfing viele Bürger Pataras in ihrem eigenen Haus ${ }^{89}$.

Auch in Ephesos sind uns - vor allem aus epigraphischen Quellen - etliche Frauen als Wohltäter (euergetes), Gymnasiarchen und Kaiserpriester ${ }^{90}$ bekannt $^{91}$, z. B. Claudia Trophime ${ }^{92}$, Tullia $^{93}$, Scholasticia ${ }^{94}$, die Vedierinnen ${ }^{95}$. Die Ephesierinnen betrachteten wohl ebenso wie ihre südkleinasiatischen 'Schwestern' die Stadt als eine erweiterte Familie, als einen »überdimensionalen oikos«, wie J. Nollé es in einem treffenden Bild beschreibt ${ }^{96}$, in dem sie ihre Pflichten ausübten und entsprechende Würden empfingen.

keine Attribute; J. C. Eule, Hellenistische Bürgerinnen aus Kleinasien. Weibliche Gewandstatuen in ihrem antiken Kontext (2001). - Die außerhalb des Dodekathlos stehende Erzählung von Herakles bei Omphale, in Lydien angesiedelt, gewinnt im Laufe der römischen Kaiserzeit an Beliebtheit und wird nicht mehr ausschließlich als Beispiel für machthungrige, männerbestimmende östliche Frauen verwendet; Herakles tauscht bildlich Löwenfell und Keule mit Spindel und Rocken; J. Nollé, Frauen wie Omphale? Überlegungen zu politischen Ämtern von Frauen im kaiserzeitlichen Kleinasien, in: Dettenhofer, Männersache 229-259; B. Wagner-Hasel, Herakles und Omphale im Rollentausch: Mythologie und Politik in der Antike, in: H. Wunder - G. Engel (Hrsg.), Geschlechterperspektiven (1998) 205-228; P. Zanker, Eine römische Matrone als Omphale, RM 106, 1999, 119-131; E. Trinkl, »Die spinnen, die Römerinnen«, in: Altmodische Archäologie. Festschrift F. Brein, Forum Archaeologiae 14/III/2000 (http:// farch.net); H. Schulze, Held in Frauenkleidern - Herakles bei Omphale, in: Herakles/Herkules (2003) 256-261. Auch das in der römischen Kaiserzeit beliebte Motiv Achilleus auf Skyros wird durch Rocken und Spindel in der Hand des Helden verdeutlicht.

87 Nollé (Anm. 86). Ähnlich argumentiert auch M. H. Dettenhofer, Frauen in politischen Krisen, in: Dettenhofer, Männersache 133-157: Einflußreiche Frauen, auch jene des Herrscherhauses, hatten die Funktion einer »Platzhalterin für einen Mann«. Vergleichbar auch P. Herz, der Frauen ein Amt nur kraft des Amtes des Ehegatten zugestehen will; P. Herz, Asiarchen und Archiereiai, Tyche 7, 1992, bes. 101: »Man scheint dabei wirklich in der Regel angestrebt zu haben, einem verheirateten Kandidaten als archiereus, sacerdos oder flamen zu wählen. « Herz gibt auf Basis der Inschriften eine Auswahl von flaminicae und weiblicher sacerdotes; ebenda $112 \mathrm{ff}$. - Zu Recht weist T. Scheer darauf hin, daß die Übernahme städtischer Ämter, die in der römischen Kaiserzeit stark an Bedeutung verloren hatten, in erster Linie das Ergebnis familiärer Umstände und nicht des Geschlechtes ist; Scheer (Anm. 78) 143-172 bes. $157 \mathrm{ff}$.

88 Okland (Anm. 1) $133 \mathrm{f}$.

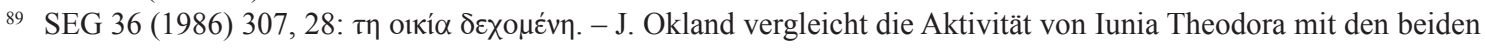
Frühchristinnen Priska und Phoibe; Okland (Anm. 1) 133 ff. s. auch E. Wallinger, Reiche Christinnen im römischen Reich, in: Specht (Anm. 75) 221-274; sie stellten der Gemeinde auf ihre eigenen Kosten einen Versammlungsplatz zur Verfügung.

90 D. Knibbe, Der Staatsmarkt. Die Inschriften des Prytaneions. Die Kureteninschriften und sonstige religiöse Texte, FiE IX 1, 1 (1981).

91 G. M. Rogers, The Construction of Women at Ephesos, ZPE 90, 1992, 215-223. Kleinasien stellt in der frühen Kaiserzeit ein besonderes Zentrum für das Stiftungswesen dar; H. Taeuber, Stifterinnen im griechischen Osten, in: Specht (Anm. 75) 199-219. Finanzielle Beiträge von Frauen sind bereits auf der sog. großen Spenderliste der späten Republik bzw. frühen Kaiserzeit verzeichnet; H. Engelmann, ÖJh 69, 2000, 79; IvE 1687.

92 IvE 1062; Knibbe (Anm. 90) 124; Merkelbach - Stauber (Anm. 86) 322 f.

93 IvE 1063; Merkelbach - Stauber (Anm. 86) 324 f.; Tullia hat »ihren Reichtum zu jedem guten Zweck reichlich verwendet $\ll$.

94 Scholastikia: IvE 453; Merkelbach - Stauber (Anm. 86) 318.

95 F. Kirbihler, Les notables d'Ephese. Essai d'histoire sociale (133 av. J.C. - 262 ap. J.C.) (ungedr. Diss. Tours 2003) $787 \mathrm{ff}$.

96 Nollé (Anm. 86) 258. 
Aber abgesehen von diesen Leistungen für die und in der Öffentlichkeit sieht sich die römische Frau als ehrenhafte verheiratete Ehefrau mit einer Fülle weiterer Aufgaben konfrontiert, die im persönlichen und privaten Umfeld wahrzunehmen sind. Sie spielen automatisch bei dem Begriff der matrona oder domina herein ${ }^{97}$. Eine dieser Pflichten ist die Verarbeitung von Wolle bzw. die Beaufsichtigung der Dienerschaft bei diesen Arbeiten ${ }^{98}$.

\section{Repräsentation am Grab}

Grabbeigaben werden traditionell und mit guten Gründen mit dem Leben des Verstorbenen in Zusammenhang gebracht. Und ebenso erfolgt die Wahl der Inschriften und Abbildungen auf Grabsteinen oder Grabmonumenten keinesfalls zufällig: Sie steht einerseits in lang geübter sozialer und kultureller Tradition, zeigt andererseits Anklänge an individuelle Züge und kann sich konkret auf das Leben des Verstorbenen beziehen. Obgleich diese Entwicklungen unabhängig von Stand und Vermögen sind, sind sie für uns selbstverständlich besser - auf Grund der größeren Materialfülle - an reichen Gräbern zu beobachten ${ }^{99}$.

Bereits M. L. Cremer erkannte, daß die auf Grabsteinen abgebildeten Spinngeräte, Spindel und Rocken, »im Sinne von kennzeichnenden Attributen beigefügt « ${ }^{100}$ sind $^{101}$; vergleichbare Erklärungen sind auch für die auf den Grabsteinen von der Verstorbenen selbst gehaltenen Spinngeräte anzuführen ${ }^{102}$. Bildlich deutlich umgesetzt sind diese Vorstellungen auf einem Jahreszeitensarkophag in Aphrodisias (Abb. 16) ${ }^{103}$ : Auf der Frontseite ist eine Familie dargestellt; der

${ }^{97}$ Als äußeres Zeichen trägt sie bis in das 3. Jh. die stola, bis Ende des 3. Jhs. ist die Bezeichnung $\mu \alpha \tau \rho \hat{\omega} v \alpha$ $\sigma \tau 0 \lambda \hat{\alpha} \tau \alpha$ gebräuchlich. B. Holtheide, Matrona stolata - femina stolata, ZPE 38, 1980, 127-131; J. Lynn Sebesta, Symbolism in the Costume of the Roman Woman, in: dies. - L. Bonfante (Hrsg.), The World of Roman Costume (1994) 46-53 bes. 48 ff. Zum auf- und abflauenden Interesse an der stola in der römischen Kaiserzeit vgl. bes. B. I. Scholz, Untersuchungen zur Tracht der römischen matrona, Arbeiten zur Archäologie (1992) 75 ff.

98 Besonders umfangreich sind die Quellen zur Wollverarbeitung zusammengetragen bei Krause (Anm. 73 ) $130 \mathrm{ff}$. Es gilt allerdings die Fälle zu trennen, in denen die Wollarbeit als belehrendes Exempel gebraucht wird und in denen sie den tatsächlichen Lebensumständen entspricht bzw. der Lebensunterhalt damit verdient werden kann (z. B. Verg. Aen. 8, 407 ff. im Gegensatz zu Anth. Gr. 6, 247). Vgl. Eichenauer (Anm. 77) 90 ff.

99 Spinngeräte werden bereits im 2 Jt. als Grabbeigaben verwendet; K. Balfanz, Eine spätbronzezeitliche Elfenbeinspindel aus Troia VIIA, Studia Troica 5, 1995, 107-116. Diese Tradition setzt sich bis in die römische Zeit fort; Trinkl, Set; Gottschalk (Anm. 22) 483-500.

${ }^{100}$ Cremer, Venuskunkeln 139 ff.: Der Kalathos ist meist ein Attribut junger Mädchen. Grabstein der Menophila aus Sardes; Merkelbach - Stauber (Anm. 86) 408 f. mit Lit.

${ }^{101}$ Pfuhl - Möbius, bes. 542; M. Waelkens, Die kleinasiatischen Türsteine (1986); J. C. Balty, Porträt und Gesellschaft in der römischen Welt, TrWPr 11 (1991) bes. 20. - Die verschiedenen Symbole (Spinnutensilien und Wollkorb) können einander auch ergänzen; z. B. J. Nollé, Grabepigramme und Reliefdarstellungen aus Kleinasien, ZPE 60, 1985, 117-135 Taf. 8b; vgl. ILS 8393. 8394; D. Flach, Die sogenannte Laudatio Turiae, Texte zur Forschung 58 (1991).

102 Welche Lösungen für die von der Verstorbenen gehaltenen Spindel und Rocken gefunden wurden, ist jedoch abhängig von zeitlichen und geographischen Entwicklungen. Ein bekanntes Beispiel ist das Grabmal der Familie des Kelten Blussus aus Mainz aus der frühen Kaiserzeit: Blussus hält einen Geldbeutel und Menimanae Rocken und Spindel; S. Böhme-Schönberger, Das Mainzer Grabmal von Menimane und Blussus als Zeugnis des Romanisierungsprozesses, in: Hüssen (Anm. 15) 1-11. Y. Freitag, Die Grabmäler der gallo-römischen Kultur im Moselland, JbRGZM 44, 1997, bes. 316 Abb. Trev 136; Out of Rome (Anm. 22) 197 Nr. 160: Grabstein eines Ehepaars aus einer Nekropole bei Aquincum. V. M. Hope, Words and Pictures: the Interpretation of Roman-British Tombstones, Britannia 28, 1997, bes. 252 Taf. 14B: die Freigelassene Regina wird - vielleicht von einem Palmyrener Steinmetzen - als 'römische matrona' auf ihrem Grabstein dargestellt. A. Schmidt-Colinet - A. Stauffer - K. Al-Asad, Die Textilien aus Palmyra, DaF 8 (2000) 50 ff.: Die Frauen auf den Grabsteinen des 2./3. Jhs. in Palmyra halten Rocken und Spindel. - Vgl. auch das Mosaik aus Edessa/Urfa mit der Darstellung einer Familie; hier tragen die Mutter und die Tochter der Hauptfigur einen Rocken; J. B. Segal, New Mosaics from Edessa, Archaeology 12/3, 1959, bes. $150.154 \mathrm{f}$.

${ }^{103}$ G. Koch - H. Sichtermann, Römische Sarkophage, HdArch V (1982) 529 Nr. 519. M. George, Family and familia on Roman Biographical Sarcophagi, RM 107, 2000, 191-207. 
Mann sitzt rechts, eine Schriftrolle haltend; links steht eine Frau, wohl eine Amme oder Sklavin, die der annähernd in der Mitte stehenden Frau ein Kind reicht. Die Ehefrau hält Spindel und Rocken in der linken Hand.

Eine Grabinschrift des 2. Jahrhunderts in Klaudiopolis lobt die Verstorbene Tertulla als Wohltäterin der Stadt und deren Bewohner. Sie nennt persönliche Eigenschaften der Tertulla, wie Milde und eine schöne Stimme, und bezeichnet die Verstorbene als besonnene Hüterin des Hauses, oíkoupò $\sigma \alpha o ́ \phi \rho \omega v^{104}$.

Grabbeigaben wie KatNr. $10^{105}$ untermauern die auf den Grabsteinen beschriebenen oder durch Gegenstände symbolisierten Tugenden der Verstorbenen ${ }^{106}$.

\section{Schlußfolgerungen: Prunkrocken als Statussymbol für die domina}

Mit den hier vorgestellten Exemplaren vergleichbare Funde stammen aus weiten Bereichen des Imperium Romanum; eine gewisse Fundlücke scheint sich derzeit auf der Iberischen Halbinsel, in Frankreich und im westlichen Nordafrika abzuzeichnen. Dies dürfte m. E. eher mit dem Forschungsstand als mit der tatsächlichen Verbreitung dieser Objektgruppe zusammenhängen. Es fällt jedoch auf, daß sehr viele vergleichbare Spinnrocken ebenso wie KatNr. 10 aus sepulkralen Kontexten stammen ${ }^{107}$, falls überhaupt Informationen zu den Fundumständen vorliegen ${ }^{108}$. Bei den Exemplaren aus dem Hanghaus 2, KatNr. 1-9, ist dies jedoch nicht der Fall. Dieser Fundort setzt verzierte Spinnrocken dieser Art eindeutig in den römischen Alltag ${ }^{109}$. Mit großer Wahrscheinlichkeit ist eine vergleichbare Herkunft aus Wohnhäusern auch für die frag-

${ }_{104}$ W. Peek, Grabepigramme aus Klaudiopolis, ZPE 27, 1977, 275-282; Inschriften griechischer Städte aus Kleinasien 31 (1986) Nr. 71.

${ }^{105}$ Cremer, Fingerkunkel, bes. 328. - Ausschließlich transzendent und als Anspielung auf die Parzen werden die Rocken als Grabbeigaben, besonders jene aus Gagat und Bernstein, von Pirling (Anm. 35) 101-109 verstanden. Ausgehend von Grabfunden in Aquileia untersucht auch D. Cottica die symbolische Bedeutung von Spinngeräten in Gräbern; D. Cottica in: Gillis - Nosch (Anm. 33).

${ }^{106}$ In der Sepulkralplastik wird deutlich, daß im Laufe der römischen Kaiserzeit Hera/Iuno als traditionelle Beschützerin der Ehe von Aphrodite/Venus abgelöst wird; M. L. Hänninen, Iuno Regina and the Roman matrons, in: P. Setälä (Hrsg.), Female networks and the public sphere in Roman society, ActaInstRomFin 22 (1999) 39-52; E. d'Ambra, The Cult of Virtues and the Funerary Relief of Ulpia Epigone, Latomus 48, 1989, 392-400: Die Verstorbene wird als Venus dargestellt. - Reiche Römerinnen identifizieren sich mit der körperlichen Schönheit der Venus und lassen Statuen anfertigen, die auf dem makellosen Körper der Göttin ein Individualporträt tragen; A. Dierichs, Erotik in der Bildenden Kunst der Römischen Welt, in: Späth - Wagner-Hasel, Frauenwelten, bes. 400. In diesen Rahmen fügt sich die Verzierung eines Gegenstandes, der den Stand und Status der domina repräsentiert, mit Venus pudica besonders gut ein. - Zur Weiterentwicklung des Motivs der Venus pudica vgl. N. Salomon, Die Venus Pudica: Eine Offenlegung der verborgenen Interessen und gefährlichen Genealogien der Kunstgeschichte, in: Wunder - Engel (Anm. 86) 79-104.

${ }^{107}$ Fingerkunkeln aus Gräbern: König, Fingerkunkel; Biró, Goddess; Ä. Kloiber, Die Gräberfelder von Lauriacum. Das Espelmayrfeld, FiL 8 (1962) 77 Grab 84 Taf. 29; Grab in Niederösterreich: Ch. Farka, Römerzeitliche Gräber aus Haselbach in Niederösterreich, RÖ 3, 1975, 79 f. Abb. 5, 3. Davidson, Corinth Nr. 2389. H. Buschhausen, Die spätrömischen Metallscrinia und frühchristliche Reliquiare, WBS 9, 1971, 78 ff. Im Museum in Piräus befindet sich eine bisher unpublizierte Fingerkunkel (freundliche Mitteilung Y. Spantidaki); wahrscheinlich aus Gräbern dürften auch die zahlreichen unterschiedlich gestalteten, im Museum von Afyon aufbewahrten Kunkeln stammen (freundliche Mitteilung A. İlasl1). Vermutlich aus Gräbern: S. Ivčević, Koštane preslice s prikazom Afrodite iz Arheološkog Muzeja Split, OpArch 23/24, 1999/2000, 473-480.

${ }^{108}$ Fingerkunkeln ohne exakte Fundortangaben bzw. aus Kunsthandel: Cremer, Venuskunkeln; MuM Sonderliste O (1972) Nr. 79 f. 82 f.; Gorny \& Mosch, Kunst der Antike 128. Auktion 13. Dez. 2003 (2003) 101 Nr. 455. - Angeblich aus Ägypten: Romans \& Barbarians, Museum of Fine Arts Boston (1977) 30 Nr. 38. - Wahrscheinlich aus Griechenland: H. Zabehlicky in: W. Melchart, Antike Kostbarkeiten aus österreichischem Privatbesitz (1997) 173 Abb. 219. - Keine genauen Fundumstände sind bei den Venuskunkeln aus Pessinus und Kertsch zu eruieren: Gomolka-Fuchs, Spinngeräte.

${ }^{109}$ Fingerkunkeln aus Siedlungen: Limeskastell Iatrus: Gomolka-Fuchs, Spinngeräte. Diogentia: erwähnt bei Gomolka-Fuchs, Spinngeräte. 
mentarischen Exemplare KatNr. 11-13 anzunehmen. Deren Fundort, unabhängig von den entsprechenden Kontexten, liegt am Nordrand des sog. Staatsmarktes von Ephesos, nahe eines der ephesischen Stadtzentren, fernab jeder römischen Nekropole ${ }^{110}$.

Wie oben gezeigt wurde, ist die Form der hier vorgestellten Artefakte von einer Fingerkunkel, einem am unteren Ende mit einer Ringöse versehenen Spinnrocken, abzuleiten. Die tatsächliche Gestalt hat sich allerdings in ihren Proportionen und in ihrer dekorativen Ausfertigung mit spitzen Fortsätzen, geschnitzten Verzierungen und bekrönenden Statuetten so weit von einem Arbeitsgerät entfernt, daß die Spinnrocken KatNr. 1-15 jedwede Praxistauglichkeit verloren haben; als Werkzeug sind sie in dieser Form vollkommen unbrauchbar. Ihre Existenz muß daher andere Erklärungsmodelle als eine praktische Berufsausübung bemühen. Gestalt und Form sind zwar von der Erzeugung eines Fadens aus der Rohwolle abzuleiten, die Bedeutung liegt jedoch in einem übertragenen Sinn; die verzierten Spinnrocken sind als Prunkrocken

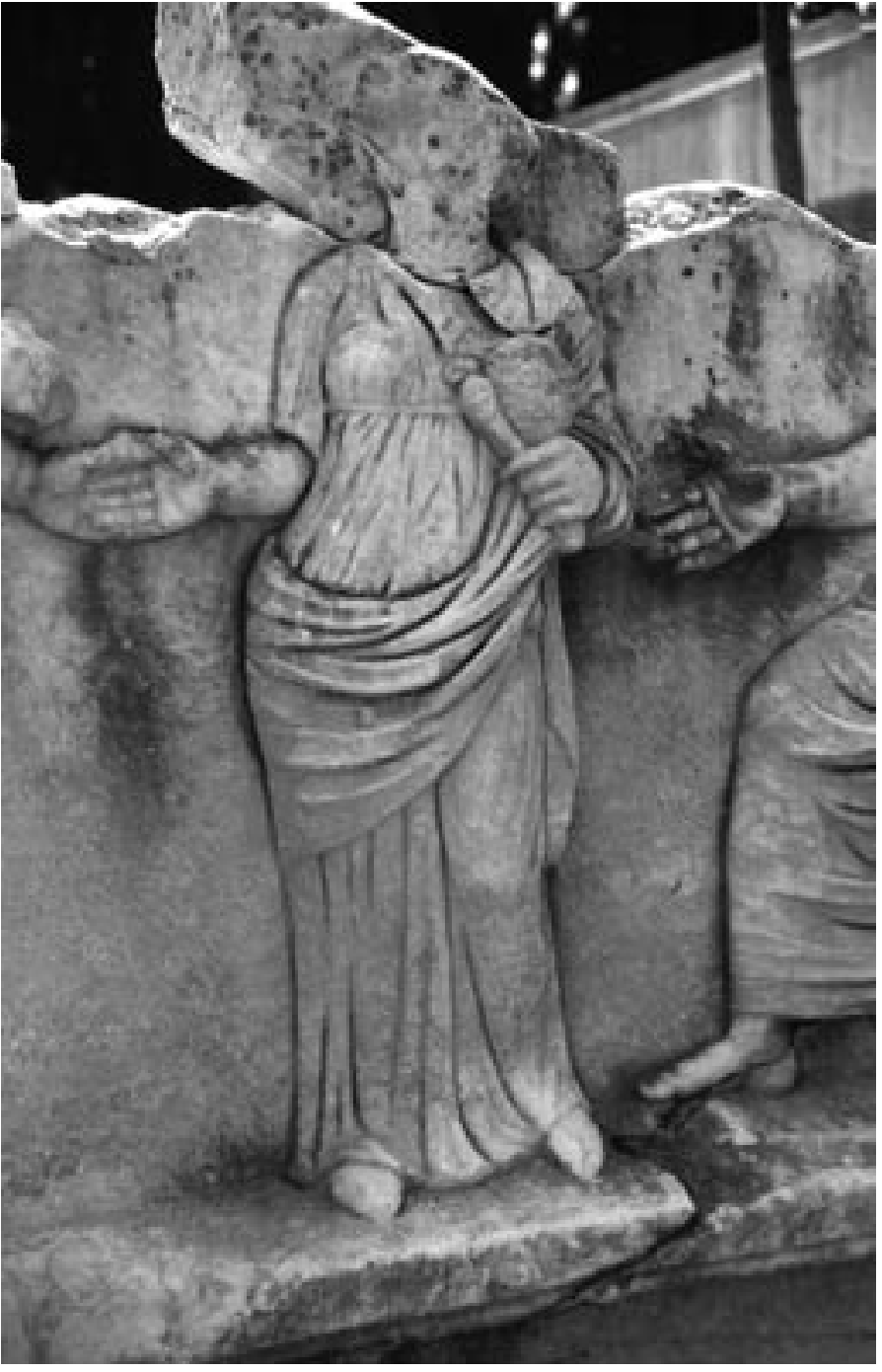

16 Jahreszeitensarkophag in Aphrodisias, Detail. Domina mit Rocken und Spindel zu verstehen ${ }^{111}$.

Das Spinnen zählt zu den tra-

ditionellen Aufgabengebieten von Frauen. In gutsituierten Haushalten wird diese Pflicht insbesondere von der Hausfrau/Ehefrau, domina, durch die Überwachung des Arbeitsprozesses wahrgenommen; die eigenhändige Produktion von Textilien, wie beispielsweise für Livia belegt, stellt eher eine Ausnahme dar, wird aber literarisch und epigraphisch als beispielgebendes und moralisierendes Exempel herausgestellt ${ }^{112}$. Das Aufgabengebiet der domina ${ }^{113}$ umfaßte jedoch weitaus vielfältigere Pflichten. Deren Kern ist der Schutz und die Bewahrung des Haushaltes, der ver-

${ }^{110}$ Der Südabhang des Panayırdağ trug wahrscheinlich den Hanghäusern 1 und 2 vergleichbare repräsentative Wohnhäuser, deren Erhaltungszustand jedoch bei weitem nicht an die Häuser am Nordhang des Bülbüldağ heranreicht; V. M. Strocka, Grabungen in Ephesos von 1960-1969 bzw. 1970. Wandmalerei, ÖJh 50, 1972-75, Beibl. 469.

${ }^{111}$ Gegenständen aus Bein werden gebleicht, poliert oder verfärbt, um ihnen einen höheren Prestigewert zu geben und sie als aus Elfenbein gefertigt ausgeben zu können. Dies wird vor allem bei Schreibgeräten und Fingerringen, aber auch bei Scharnieren angewendet und könnte auch bei unseren Prunkrocken der Fall gewesen sein; E. DeschlerErb, Römische Beinartefakte aus Augusta Raurica, Forschungen in Augst 27/1 (1998) $81 \mathrm{ff}$.

${ }^{112}$ Col. 12 pr.; Suet. Aug. 74. Livia wird in die Propaganda als Repräsentantin der Domus Augusta einbezogen; A. Dierichs, Das Idealbild der römischen Kaiserin: Livia Augusta, in: Späth - Wagner-Hasel, Frauenwelten 241-262.

${ }^{113}$ Das von Vitruv tradierte Bild der spinnenden Frauen im oecus zeigt nur einen kleinen Teil der weiblichen Aufgabengebiete; Vitr. 7, 149, 2. 
schiedenartige familiäre, wirtschaftliche und soziale Belange einschließt. Die öffentliche Leitung des Haushaltes liegt beim patronus/dominus, die interne jedoch bei der matrona/domina. Das tatsächliche Wohnhaus, domus, steht auch symbolisch für den gesamten Haushalt.

Bereits im Rahmen des Abschnitts über die sepulkrale Symbolik wurde kurz auf die verschiedenartigen Fähigkeiten von Frauen eingegangen. Darauf möchte ich kurz zurückkommen. Frauen, wie sie uns hier aus dem Hanghaus 2 in Ephesos als wahrscheinliche Besitzerinnen der Prunkrocken entgegentreten, gehörten auch bezüglich ihrer Bildung zur Elite. Ebenso wie die Bildung des Hausherren in den Bibliotheken und durch die Musenzimmern zur Schau gestellt wird ${ }^{114}$, kann die Hausherrin ihre Bildung und Fähigkeiten besonders an einem Gegenstand wie der, leider nur fragmentarisch erhaltenen, Fingerkunkel KatNr. 2 demonstrieren. Wenn in Anlehnung an von mit Minervabüsten bekrönte gallische Wachsspateln ${ }^{115}$ die Interpretation der den Spinnrocken bekrönenden Scheibe als Brust einer Minervabüste zutrifft, werden in der Fingerkunkel KatNr. 2 zwei Hauptaspekte der lateinischen domina verbunden: die Herrschaft über das Haus, veranschaulicht durch die Wahl der Form eines Spinnrockens, wird durch eine entsprechende Ausbildung und Bildung, symbolisiert durch die Minervabüste, ermöglicht.

Mit der Hochzeit übernimmt die Römerin als domina ihre Pflichten gemäß ihrer sozialen Stellung in der domus ihres Ehemannes, ohne jedoch ihre finanzielle Eigenständigkeit einzubüßen. Und gerade ein von Plinius überlieferter Brauch im Rahmen der Hochzeitszeremonie gibt uns den Schlüssel zum Verständnis der Prunkrocken: »Daher der Brauch, daß Jungfrauen bei der Hochzeitsfeier ein geputzter Rocken und eine Spindel nebst Faden begleiteten. $\ll^{116}$ Auch Plutarch bestätigt, daß die Braut bei der Hochzeit Rocken und Spindel trägt ${ }^{117}$.

So scheint es mir naheliegend, daß die hier vorgestellten Prunkrocken aus dem Hanghaus 2 in Ephesos ursprünglich als Hochzeitsgeschenk in den Besitz der domina gelangten. Sie bewahrte den dekorierten Spinnrocken auf, und mancher Römerin wurde ihr Hochzeitsgeschenk als Beigabe ins Grab mitgegeben. In der Zwischenzeit stellte die domina/matrona den Prunkrocken als Attribut ihrer häuslichen Autorität in einem öffentlich zugänglichen Bereich der domus zur Schau.

114 N. Zimmermann, Ausstattung von Haupt- und Nebenräumen. Zur Datierung der Wandmalereien des Hanghauses 2 in Ephesos, in: Krinzinger, Baugeschichte 101-117. H. Thür, Privatbibliotheken im Hanghaus 2 in Ephesos, in: Asamer - Wohlmayr (Anm. 54) 205-210.

${ }^{115}$ Feugère (Anm. 15) 328 Nr. 19 Abb. 6c. Die Verwendung der Göttin Minerva im Umfeld des Schriftwesens als 'inspiratrice de l'intelligence' ist naheliegend; Minerva als Zeichen der Bildung der domina auf einem Rocken erscheint mir auf eine vergleichbare Weise verständlich. - Vgl. die von J. Fabricius herausgestrichenen Symbole der Bildung auf rhodischen Grabsteinen der hellenistischen Zeit; Fabricius (Anm. 86).

${ }^{116}$ Plin. nat. 8, 194. Plinius erwähnt diesen Brauch im Zusammenhang mit Herkunft und Aussehen des Hochzeitsgewandes: inde factum, ut nubentes virgines comitaretur colus compta et fusus cum stamine. In diesem Zusammenhang würde auch der auf einem Silberkalathos aus Xanten von einem kleinen Knaben getragene Gegenstand verständlich; viel eher als ein Alabastron oder ein Gefäß anderer Form scheint mir in diesem 'Liebes- oder Hochzeitsbild' ein beladener Rocken oder eine Spindel, die wegen des sich auf ihr befindenden Fadens in der Mitte verdickt ist, dargestellt zu sein. D. Grassinger, Iason und Kreusa? Zur Deutung der Szene des Silbercalathus aus WardtLüttingen, in: H. H. von Prittwitz und Gaffron (Hrsg.), Das Haus lacht vor Silber (1997) 125-138; M. Menninger, Die römischen Silbergefäße im Rheinischen Landesmuseum Bonn, in: ebenda, bes. 99 ff. Abb. 1 ff. Die Form des Gefäßes stellt eine weitere Beziehung zum Aufgabenprofil von Ehefrauen her. - Nach dem Brauch stellt die Braut ihr Hochzeitsgewand selbst her; Sebesta (Anm. 97) 46-53. Eine mögliche Verbindung der Kunkeln mit der Eheschließung wurde bereits mehrfach angedacht: Gomolka-Fuchs, Spinngeräte, bes. 113. Sie verweist auch auf den noch im 19. Jh. in Rumänien üblichen Brauch, daß der Bräutigam der Braut einen Spinnrocken als Hochzeitsgeschenk überreicht. Cremer, Venuskunkeln. Vgl. Wasowicz (Anm. 26) 268-272. - Nach einem Papyrus aus der Zeit

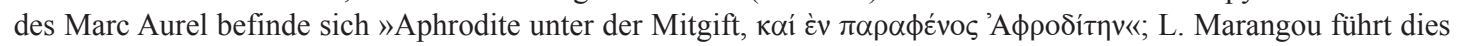
als mögliche Erklärung für die häufig auf zu Kästchen (Hochzeitsgeschenken?) gehörenden Beinplättchen dargestellte Venus an; L. Marangou, Bone Carvings from Egypt (1976) 39 ff. Man könnte in diesem Zusammenhang jedoch auch an glatte, mit Venusstatuetten verzierte Haarnadeln (E. Trinkl, Bemerkungen zu kleinasiatischen Venuskunkeln, Bulletin Instrumentum 15, 2002, 31-35; E. Hudeczek, Kleine Schmuckstücke mit großen Vorbildern, JberJoanneum N. F. 23, 1994, 69-77) oder eben auch an Venuskunkeln denken.

117 Plut. mor. 271 f. - Theokr. 17, 34 ff. Cremer, Venuskunkeln 141: »Aphrodite fungiert als Schirmherrin der Hochzeit par excellence«. Zu Frauendarstellungen auf Nadeln allgemein s. T. M. Bíró, Bone-carvings from Brigetio in the collection of the Hungarian National Museum, ActaArchHung 39, 1987, 176 ff. Abb. 16. 


\section{Abgekürzt zitierte Literatur}

Bíró, Goddess

Cremer, Venuskunkeln

Cremer, Spinnrocken

Cremer, Fingerkunkel

Davidson, Corinth

Dettenhofer, Männersache

Dickmann, Domus

Gomolka-Fuchs, Spinngeräte

IvE

König, Fingerkunkel

Krinzinger, Baugeschichte

Krinzinger, Wohneinheiten

Ladstätter, Chronologie

Lang-Auinger, Hanghaus

Larrson Lovén Strömberg, Women

Späth - Wagner-Hasel, Frauenwelten Thür, Hanghaus

Treggiari, History

Trinkl, Set

Trinkl, Artefakte

Waldbaum, Sardis
M. T. Bíró, The Unknown Goddess of Late Roman Popular Religious Belief, ActaArchHung 46, 1994, 195-229.

M. L. Cremer, Venuskunkeln aus Kleinasien, AA 1996, 135-144.

M. L. Cremer, Antike Spinnrocken, Boreas 19, 1996, 241-245.

M. L. Cremer, Fingerkunkel und Zettelstrecker. Neuzugänge im Archäologischen Museum, Boreas 21/22, 1998/99, 327-332.

G. R. Davidson, The minor objects, Corinth XII (1952).

M. H. Dettenhofer (Hrsg.), Reine Männersache? Frauen in Männerdomänen der antiken Welt (1994).

J. A. Dickmann, domus frequentata. Anspruchsvolles Wohnen im pompejanischen Stadthaus, Studien zur antiken Stadt 4 (1999).

G. Gomolka-Fuchs, Spätrömische-frühbyzantinische Spinngeräte, in: K. Kuzmová K. Pieta - J. Rajtár (Hrsg.), Zwischen Rom und dem Barbaricum. Festschrift T. Kolník (2002) 111-116.

Inschriften griechischer Städte aus Kleinasien 11, 1 (1979) - 17, 4 (1982); Ephesos (IvE Ia-VII 2).

G. G. König, Die Fingerkunkel aus Grab 156, in: K. Roth-Rubi - H. R. Sennhauser (Hrsg.), Römische Straßen und Gräber, Verenamünster Zurzach I (1987) 129-141.

F. Krinzinger (Hrsg.), Das Hanghaus 2 von Ephesos. Studien zu Baugeschichte und Chronologie, AForsch 7 (2002).

F. Krinzinger (Hrsg.), Das Hanghaus 2 in Ephesos. Die Wohneinheiten 1 und 2. Baubefund, Ausstattung, Funde, FiE VIII 7 (in Druckvorbereitung).

S. Ladstätter, Die Chronologie des Hanghauses 2, in: F. Krinzinger (Hrsg.), Das Hanghaus 2 von Ephesos. Studien zu Baugeschichte und Chronologie, AForsch 7 (2002) $9-40$.

C. Lang-Auinger (Hrsg.), Das Hanghaus 1 in Ephesos. Ausstattung und Funde, FiE VIII 4 (2003).

L. Larrson Lovén - A. Strömberg (Hrsg.), Aspects of Women in Antiquity. Proceedings of the First Nordic Symposium on Women's Lives in Antiquity, SIMA pocket book 153 (1998).

Th. Späth - B. Wagner-Hasel (Hrsg.), Frauenwelten in der Antike. Geschlechterordnung und weibliche Lebenspraxis (2000).

H. Thür (Hrsg.), Das Hanghaus 2 in Ephesos. Die Wohneinheit 4. Baubefund, Ausstattung, Funde, FiE VIII 6 (2005).

S. Treggiari, Roman Social History (2002).

E. Trinkl, Ein Set aus Spindel, Spinnwirtel und Rocken aus einem Sarkophag in Ephesos, ÖJh 63,1994, Beibl. 80-86.

E. Trinkl, Artefakte für die Textilbearbeitung, in: C. Lang-Auinger (Hrsg.), Das Hanghaus 1 in Ephesos. Ausstattung und Funde, FiE VIII 4 (2003) 313-327.

J. C. Waldbaum, Metalworks from Sardis. The Finds through 1974, Sardes VIII (1993).

\section{Dr. Elisabeth Trinkl}

c\% Österreichisches Archäologisches Institut, Franz Klein-Gasse 1, A-1190 Wien

E-Mail: elisabeth.trinkl@oeai.at

Abbildungsnachweis: Abb. 1a. b. 14: ÖAI; Abb. 2. 3. 5. 7. 11-13: ÖAW; Abb. 4a. b. 6. 15: ÖAW, Photo A. Sulzgruber; Abb. 8: Skizze Fundjournal 1963, S. 36; Abb. 9: ÖAI, Photo N. Karger; Abb. 10: Zeichnung Verf.; Abb. 16: Photo Verf. 
